\title{
Genome-wide analysis of wheat DNA- binding with one finger (Dof) transcription factor genes: evolutionary characteristics and diverse abiotic stress responses
}

Yue Liu ${ }^{1+}$, Nannan Liư ${ }^{1+}$, Xiong Deng ${ }^{1}$, Dongmiao Liu', Mengfei Li ${ }^{1}$, Dada Cui ${ }^{1}$, Yingkao Hu${ }^{1 *}$ and Yueming Yan ${ }^{1,2^{*}}$ (D)

\begin{abstract}
Background: DNA binding with one finger (Dof) transcription factors play important roles in plant growth and abiotic stress responses. Although genome-wide identification and analysis of the DOF transcription factor family has been reported in other species, no relevant studies have emerged in wheat. The aim of this study was to investigate the evolutionary and functional characteristics associated with plant growth and abiotic stress responses by genome-wide analysis of the wheat Dof transcription factor gene family.

Results: Using the recently released wheat genome database (IWGSC RefSeq v1.0), we identified 96 wheat Dof gene family members, which were phylogenetically clustered into five distinct subfamilies. Gene duplication analysis revealed a broad and heterogeneous distribution of TaDofs on the chromosome groups 1 to 7, and obvious tandem duplication genes were present on chromosomes 2 and 3.Members of the same gene subfamily had similar exon-intron structures, while members of different subfamilies had obvious differences. Functional divergence analysis indicated that type-II functional divergence played a major role in the differentiation of the TaDof gene family. Positive selection analysis revealed that the Dof gene family experienced different degrees of positive selection pressure during the process of evolution, and five significant positive selection sites (30A, 31 T, 33A, $102 \mathrm{G}$ and 104S) were identified. Additionally, nine groups of coevolving amino acid sites, which may play a key role in maintaining the structural and functional stability of Dof proteins, were identified. The results from the RNA-seq data and qRT-PCR analysis revealed that TaDof genes exhibited obvious expression preference or specificity in different organs and developmental stages, as well as in diverse abiotic stress responses. Most TaDof genes were significantly upregulated by heat, PEG and heavy metal stresses.
\end{abstract}

Conclusions: The genome-wide analysis and identification of wheat DOF transcription factor family and the discovery of important amino acid sites are expected to provide new insights into the structure, evolution and function of the plant Dof gene family.

Keywords: Wheat, Dof transcription factors, Phylogenetics, Evolution, Transcript expression, Abiotic stress

\footnotetext{
* Correspondence: yingkaohu@cnu.edu.cn; yanym@cnu.edu.cn

${ }^{\dagger}$ Yue Liu and Nannan Liu contributed equally to this work.

${ }^{1}$ College of Life Science, Capital Normal University, Xisanhuan Beilu No. 105,

100048 Beijing, People's Republic of China

Full list of author information is available at the end of the article
}

(c) The Author(s). 2020 Open Access This article is licensed under a Creative Commons Attribution 4.0 International License, which permits use, sharing, adaptation, distribution and reproduction in any medium or format, as long as you give appropriate credit to the original author(s) and the source, provide a link to the Creative Commons licence, and indicate if changes were made. The images or other third party material in this article are included in the article's Creative Commons licence, unless indicated otherwise in a credit line to the material. If material is not included in the article's Creative Commons licence and your intended use is not permitted by statutory regulation or exceeds the permitted use, you will need to obtain permission directly from the copyright holder. To view a copy of this licence, visit http://creativecommons.org/licenses/by/4.0/ The Creative Commons Public Domain Dedication waiver (http://creativecommons.org/publicdomain/zero/1.0/) applies to the data made available in this article, unless otherwise stated in a credit line to the data. 


\section{Background}

Transcription factors (TFs) involve in activating or inhibiting the activity of RNA polymerase to regulate the spatiotemporal expression of the target genes by recognizing specific DNA sequence elements present in the promoter region of the gene [1]. DNA binding with one finger (Dof) transcription factors are plant-specific. Dof proteins are generally 200-400 amino acids long with a highly conserved Dof domain of 50-52 amino acids, which is structured as a $\mathrm{C} 2 \mathrm{C} 2$-type zinc finger that recognizes a cis-regulatory element with the common core sequence of $5^{\prime}$-AAAG-3' [2-4]. Unlike the conserved $\mathrm{N}$-terminal domain, a transcriptional regulatory domain at the C-terminal of Dof proteins varies greatly, which can react with different regulatory proteins or substances to activate or inhibit gene transcription [3].

The Dof domain is a bifunctional domain that mediates both DNA-protein and protein-protein interactions $[5,6]$. The first protein-protein interaction was observed in the Arabidopsis thaliana Dof domain protein OBP1, which interacted with bZIP proteins associated with stress responses. OBPl specifically increased the binding of the OBF proteins to octopine synthase ('ocs') element sequences [7]. The Dof transcription factor prolaminbox binding factor (PBF) in maize can activate the gene expression of cereal storage protein by binding to the Pbox present in the prolamin gene (zein) promoter. Meanwhile, PBF can interact with bZIP protein Opaque2 (O2) to activate gamma-zein expression and regulate the protein content of the endosperm in maize [5]. OsDof3 in rice regulates the gibberellin response through interaction with GAMYB [8]. The Dof protein SAD from barley can activate transcription of endosperm-specific genes through interaction with R2R3MYB protein [9]. AtDof3.2 from $A$. thaliana acts as a negative regulator of seed germination and interacts with a positive regulator of seed germination TCP14 [10].

Dof TFs have important functions in plant growth and development, as well as various environmental stress responses. The function of Dof genes in A. thaliana has been extensively studied, and several AtDof genes have been shown to function in plant growth and $\mathrm{C} / \mathrm{N}$ metabolism [11, 12], shoot branching and seed coat formation [13], vascular tissue development and interfascicular cambium formation [14], photoperiodic control of flowering [15-17], morphogenesis and stomata functioning [18], and abiotic stress tolerance [17]. PBF (RPBF) Dof, an activator of seed storage protein genes in rice, participates in the regulation of endosperm-specific gene expression [19]. OsDof12 regulates flowering in long-day condition, and is inhibited by dark treatment [20]. The PBF Dof in maize is involved in seed protein and starch biosynthesis [21, 22]. ZmDof3 plays an important role in maize endosperm development [23]. Maize Dof1 can activate the PEPC gene expression and enhance transcription from the promoters of pyruvate kinase and orthophosphate dikinase [24]. Several tomato Dof genes were found to participate in the control of flowering time and abiotic stress responses [25]. In wheat, PBF homologous genes TaDof2 (WPBF-A), TaDof3 (WPBF$D)$ and TaDof6 (WPBF-B) were found to locate on the $\mathrm{A}, \mathrm{B}$ and $\mathrm{D}$ genomes of common wheat (Triticum aestivum L.), respectively [26, 27]. Wheat PBF could transactivate the transcription of the native alpha-gliadin promoter by binding to the intact prolamin-box [28]. TaDof1 was found to participate in the process of nitrogen assimilation and control the expression of genes involved in nitrogen assimilation, specifically $G S$ and GOGAT [29]. A recent report has shown that the TaDof2, TaDof3 and TaDof6 genes are involved in water-deficit response [30].

As a DNA-binding protein, the first Dof protein (ZmDof1) was identified and characterized in maize [31]. Subsequently, a large number of Dof genes have been found in different plant genomes. The number of Dof genes identified from genome-based surveys varies depending on the plant species, such as 36 in A. thaliana [3], 30 in rice [32], 26 in barley [33], 28 in sorghum [34], 27 in Brachypodium distachyon [35], 34 in tomato [36], 46 in maize [37], 36 in cucumber [38], 33 in pepper [39] and 29 in eggplants [40]. Common wheat, as an allohexaploid species, has a huge genome (up to $17 \mathrm{~GB}$ ) and more than $85 \%$ repeat sequences, leading to the slow progress of wheat genome sequencing. Earlier work only identified 31 Dof genes in bread wheat [27]. Since 2018, the wheat genome project has made great progress, and the wheat genome data have been updated to IWGSC Annotation v1.0 by the International Wheat Genome Sequencing Consortium (IWGSC) through an improvement of the current wheat chromosome level assembly [41]. The completion of the sequencing of the wheat genome will accelerate the studies on the structure, evolution and function of the wheat Dof gene family.

In this study, using common wheat genome database (IWGSC RefSeq v1.0), we conducted a comprehensive genome-wide analysis on the structural characterization, molecular evolution and expression profiling of the wheat Dof gene family, which can provide new information for further understanding the evolution characteristics and function of plant Dof genes.

\section{Results}

\section{Genome-wide identification of wheat Dof genes}

Firstly, 36 and 30 Dof protein sequences from $A$. thaliana and rice were downloaded from the PlantTFDB v4.0 database (Table S1). These sequences were used as queries for searches in the recently released Triticum aestivum (common or bread wheat) genome database (IWGSC RefSeq 
v1.0). Then SMART and Pfam websites were used to further identify whether candidate sequences have conserved Dof domain. Ultimately, a total of 96 members of the Dof transcription factor gene family were identified from wheat. For convenience, these TaDof genes were assigned names from TaDof1 to TaDof96 as listed in Table S2.

The number of amino acids of the TaDof encoding proteins varied from 152 to 539 amino acids, their pI values ranged from 4.66 to 10.46 with an average of 8.05 and weakly alkaline, and their molecular weights were from 15.77 to $58.13 \mathrm{kDa}$, with an average of $33.38 \mathrm{kDa}$. Their detailed information is shown in Table S2. These results indicated that variations in the amino acid sequence length of Dofs may be associated with adaptation to different functional requirements and physical/chemical properties.

\section{Chromosome location and genes duplication of 96 TaDof genes}

Based on the IWGSC database, the physical locations of the TaDof genes on the corresponding chromosomes are depicted in Fig. 1. All TaDof genes identified could be mapped on the chromosomes from 1A to 7D. Evidently, the TaDof genes were unevenly distributed on different chromosomes, including 34 TaDof genes in chromosome A, 32 in chromosome B, and 30 in chromosome D. Most TaDofs genes had corresponding homologous genes on the A, B, and D chromosomes. In particular, chromosome 3 with 27 TaDof members from TaDof 38 to TaDof64 had the highest density, and they were closely arranged at the lower part of the chromosomes, but chromosome 7 only contained three TaDof genes (TaDof94, TaDof95 and TaDof96). Interestingly, we found that the genes located on chromosome $4 \mathrm{~A}$ were opposite to the position of the homologous genes located on chromosome $4 \mathrm{~B}$ and chromosome 4D. In addition, segmental duplication and tandem duplication analysis revealed that TaDofs transcription factor family was not generated by segmental duplication, but obvious tandem duplication genes were present at the ends on the chromosomes 2 and 3.

\section{Subcellular localization of TaDof proteins}

The predicted cellular localizations by the five different software programs showed that all 96 TaDof proteins were located in the nucleus (Table S2). Then three TaDof proteins (TaDof2, TaDof3 and TaDof6) were chosen to further perform transient expression to verify the subcellular localization predictions. The results showed that strong green fluorescent signals of the three GFP fusion proteins were observed in the nucleus (Fig. 2), confirming that these TaDof proteins were located in the nucleus. These results are consistent with the transcription factor characteristics and the software predictions.

\section{Phylogenetic relationships and molecular characterization of TaDof transcription factors}

Multiple sequence alignments of the 162 Dof proteins were performed to construct a Bayesian phylogenetic tree (Fig. 3) and a Neighbor-joining (NJ) phylogenetic tree (Fig. S1). The trees revealed that the 96 TaDof genes in wheat were classified into five subfamilies (Group AE) based on later topology and structural similarity analysis, among which Group D was the largest branch with 28 TaDof members. Both Group B and C had 20 members, followed by Group A with 15 members and Group E with 13 members. As anticipated, the wheat phylogenetic trees constructed by the NJ method (Fig. 4a), maximum likelihood method and minimal evolution method (Fig. S2) showed a similar topological structure for the five subfamilies.

The exon-intron structures of the 96 TaDof gene members were analyzed by comparing the CDSs and the complete gene sequences using the GSDSv2.0, and the results are shown in Fig. 4b, including CDS, intron and UTR structures. The number of introns in the TaDof genes was extremely small, with 0-2 introns in each gene. Except for TaDof43 with two introns, 51 TaDof genes $(53.13 \%)$ had only one intron, and the remaining 46 TaDof genes (45.83\%) had no intron (Table S2). In addition, the members of the same subfamily generally had similar number of introns. The intron length also varied greatly among different subfamilies, likely resulting from the absence or gain of introns during longterm evolutionary processes.

To further investigate the diversity of Dof genes in wheat, the MEME program was used to analyze the potential motif composition in the Dof gene family. In total, 15 different motifs were identified (Fig. 4c and Fig. S3). Motif 1, a conserved Dof domain, was uniformly observed in all TaDof proteins. Except for individual members, the same subfamily of Dof proteins generally shared similar motif number, type and spatial arrangement, implying similar functions of Dof proteins in the same subfamily. Group A and B contained fewer motifs, almost exclusively had Motif 1 . The specific motif 15 was present in Group $C$, the conserved motif 2 was present in Group D, and the conserved motifs 4, 6, and 9 occurred in Group E. The remaining motifs 7, 8, 11, 12,13 and 14 were variable.

\section{Functional divergence analysis of TaDof transcription factors}

The DIVERGE v3.0 software combined with the posterior probability analysis method [42-44] was used to estimate the type-I and type-II functional divergences of the 


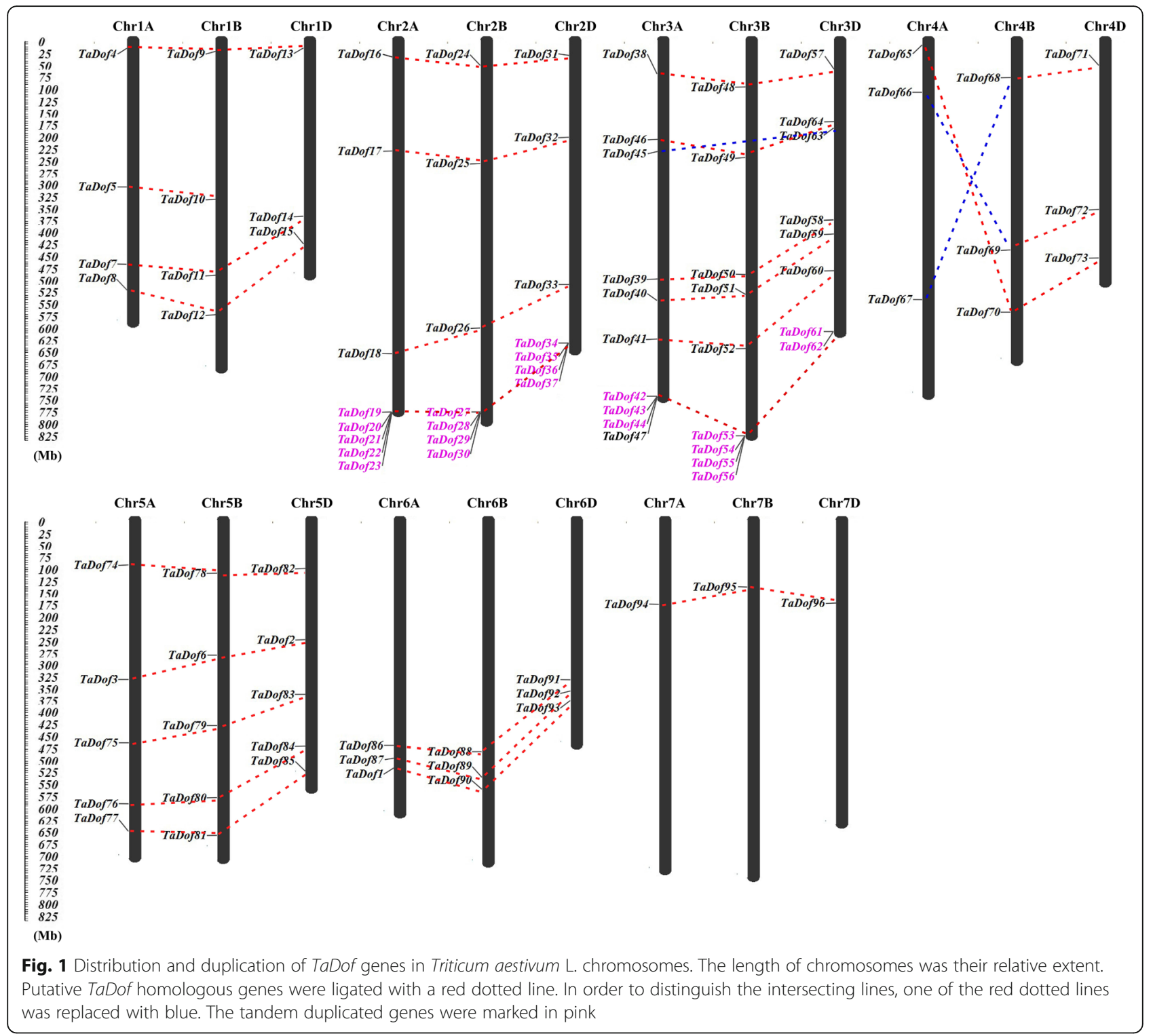

gene group in the Dof family. The results showed that, except for subfamily pairs Group A/Group B, Group A/ Group C, Group D/Group C and Group B/Group C, the type-I function divergence coefficient $\left(\theta_{\mathrm{I}}\right)$ among other groups ranged from 0.177 to 0.418 , which is significantly larger than 0. Among them, likelihood ratio test (LRT) values of the subfamily pairs Group A/Group E, Group C/Group E and Group D/Group E were significantly different $(p<0.05)$, indicating that the possible presence of type-I divergence sites during the evolution between groups of wheat Dof proteins. No significant type-I function divergence was found among other groups. Similarly, the type-II functional divergence coefficient $\left(\theta_{\mathrm{II}}\right)$ ranged from -0.157 to 0.164 , indicating that type-II functional divergence sites may also be present (Table 1).
Critical amino acid sites were identified in five groups of TaDof subfamilies in the analysis of type-I and type-II functional divergence. In this study, $\mathrm{Qk}>0.8$ was used as a threshold to screen important amino acid sites, which can reduce the occurrence of false positives. As shown in Table 1, only one type-I functional divergence amino acid site (30A) was detected between Group D and Group E, indicating that the evolutionary rate of this amino acid site might change significantly. Eleven typeII functional divergence sites were found, including $30 \mathrm{~A}$, $32 \mathrm{~A}, 33 \mathrm{~A}, 45 \mathrm{~K}, 47 \mathrm{E}, 52 \mathrm{~K}, 55 \mathrm{~N}, 66 \mathrm{M}, 71 \mathrm{Y}, 75 \mathrm{~A}$ and 94G. These may be the key amino acid sites affecting physical and chemical properties of $\mathrm{TaDof}$ proteins. Apparently, the type-II functional divergence sites were significantly more abundant than type-I functional divergence site, indicating that the type-II functional 


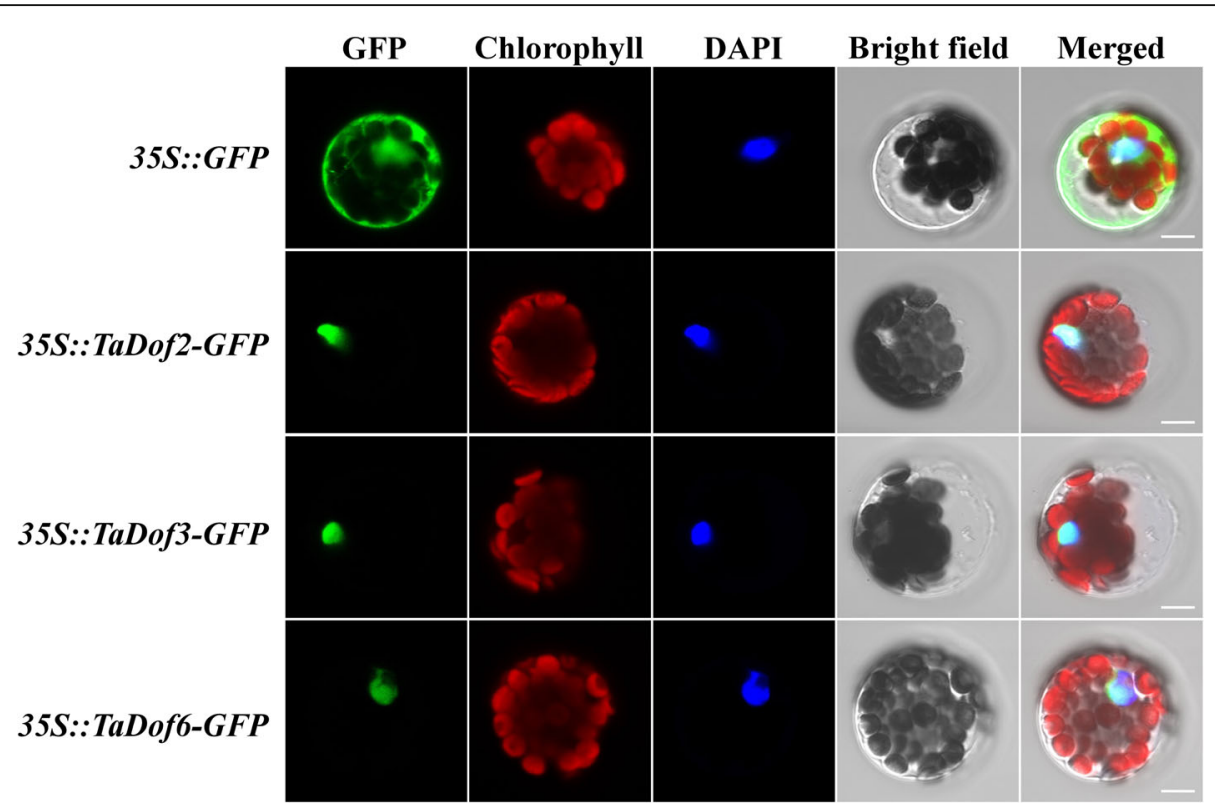

Fig. 2 Subcellular localization of wheat TaDof2, TaDof3 and TaDof6. The localization of the nuclei was detected by 4',6-diamidino-2-phenylindole (DAPI) staining. GFP: GFP fluorescence signal. Green fluorescence indicates the location of TaDofs in the Arabidopsis protoplasts; Chlorophyll: chlorophyll autofluorescence signal. Red fluorescent signal indicates the location of chloroplasts in protoplasts; DAPI: Blue fluorescence signal. Blue fluorescence indicates the location of the nucleus stained by DAPI; Bright light: field of bright light; Merged: emergence of the GFP

fluorescence signal, chlorophyll autofluorescence signal and bright light field; Nagtive: Wild-type (Clo) Arabidopsis protoplast cell. Scale bar $=5 \mu \mathrm{m}$

divergence played a major role in the differentiation of the TaDof gene family. In particular, the amino acid site $30 \mathrm{~A}$ belonged to both type-I and type-II functional divergence sites, suggesting that the evolutionary rate and physicochemical properties of this site have changed concurrently (Fig. 5a and b).

Positive selection, coevolution and three-dimensional (3D) structure analysis of TaDofs

The CODEML program in the PAML v4.4 software was used for positive selection analysis and positive selection site identification for the TaDof gene family. The site model used in this study included M0 (one scale) and M3 (discrete) as well as M7 (beta) and M8 (beta and $\omega$ ) based on the previous method [45]. By comparing M0 and M3 models, we found that the twice log-likelihood difference of the models $(2 \triangle \operatorname{lnL})$ was 883.03 , indicating that certain amino acid sites might be undergone strong positive selection pressure. Comparison between M7 and M8 models can determine whether TaDof gene family members were subjected to positive selection pressure during the evolutionary process. The results revealed that the value of $2 \Delta \operatorname{lnL}$ between the two models was 2036.983 with an extremely significant statistical difference. The estimated $\omega$ value of the M8 model was 2.55223 , which is much higher than 1 , indicating that some TaDof amino acid sites were strongly affected by positive selection. In total, 11 positive selectivity amino acid sites were detected in the M8 model, including one significant (102G, $p<0.05)$ and four extremely significant $(30 \mathrm{~A}, 31 \mathrm{~T}, 33 \mathrm{~A}, 104 \mathrm{~S}, p<0.01)$ positive selection sites (Table 2).

CAPS, a distance-sensitive coevolutionary analysis software for amino acids, was used to detect the TaDof gene family [46], and nine coevolution sites were detected (Table S3). Among them, 8 groups were adjacent in the primary structure, and most of them were distributed in different locations outside the functional structure domain (Fig. 5a).

The 3D structures of TaDof6 proteins constructed by the online software PHYRE2 showed that five significant and extremely significant sites were located on the 3D structure of TaDof6 protein (Fig. 5b-c), which were mainly located at the $\mathrm{N}$-terminal of the Dof protein. These suggest that the $\mathrm{N}$-terminal of $\mathrm{TaDof}$ proteins might encounter more positive selection pressures during the evolutionary process.

\section{Analysis of cis-acting elements in wheat Dof transcription factors}

The potential cis-acting elements in the promoter region among 96 TaDof transcription factor members were analyzed by the online tool PlantCARE, which can benefit the understanding of TaDof gene expression and function [47]. In total, seven types of cis-acting elements were found in the promoter region of the TaDof genes, as shown in Table S4. 


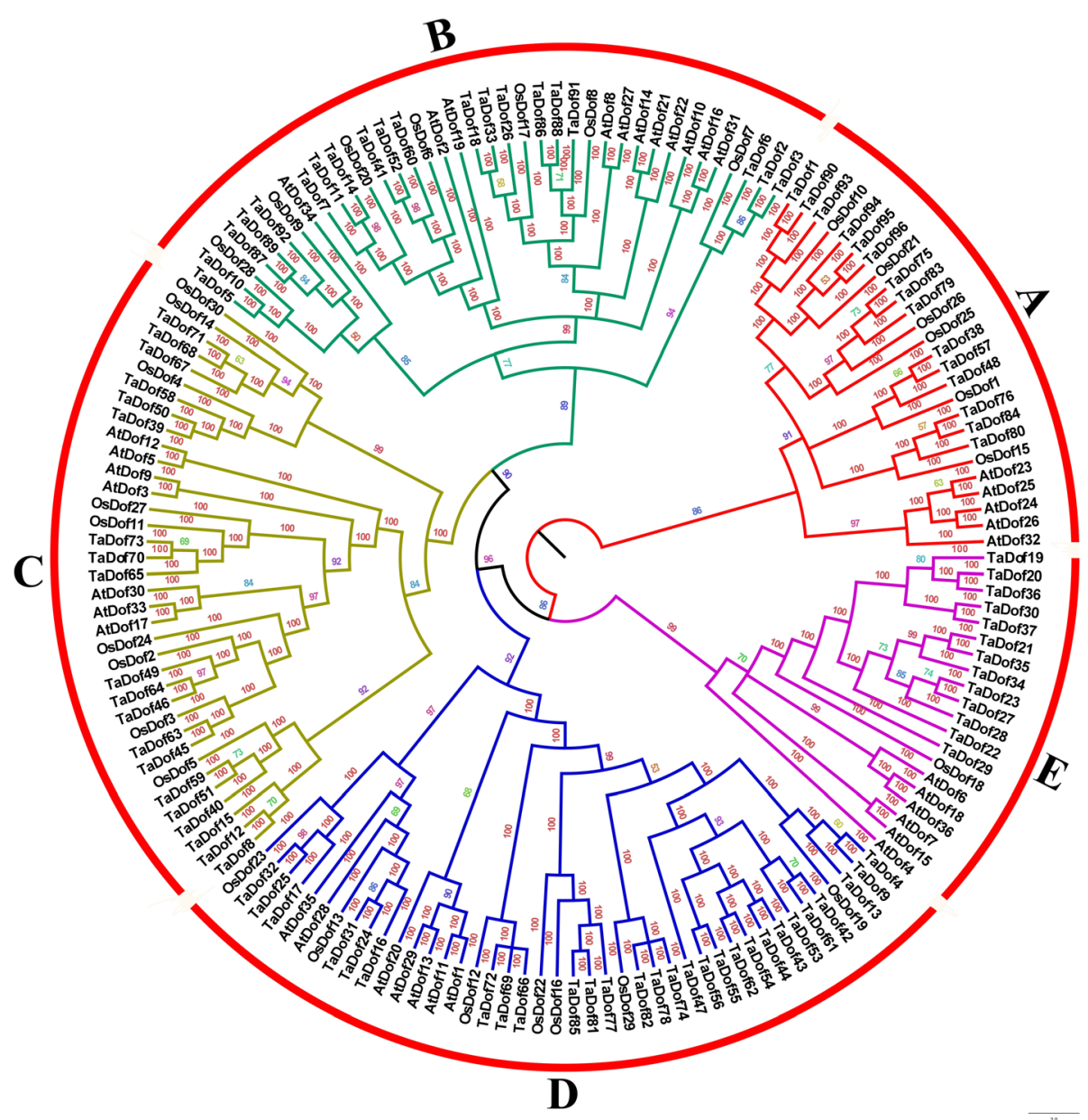

Fig. 3 The Bayesian phylogenetic tree of Dof transcription factor gene family from Triticum aestivum, Arabidopsis thaliana and Oryza sativa

Light responsive elements are a very abundant class of cis-acting elements among the TaDof gene family members, including G-box, Sp1, and Box 4. G-box seems to be the most abundant type of light responsive elements in the TaDof gene family, with a cumulative number of 263. Only 17 members of the TaDof gene family did not contain G-box while the remaining members had at least one G-box copy. Hormone responsive elements, mainly including TATC-box, GARE-motif, TCA-element, TGAelement, ABRE, TGACG-motif and CGTCA-motif, participate in response to gibberellin, salicylic acid (SA), auxin, abscisic acid (ABA) and methyl jasmonic acid (MEJA). Among them, ABRE (87.5\%), TGACG-motif (78.12\%) and CGTCA-motif (79.17\%) were present in a large number of members, with an average number of copies of 3.12, 2.67 and 2.66, respectively.

Environmental stress-related elements are also noteworthy. For instance, the GC-motif (68 copies) and ARE (108 copies), which are involved in the regulation of gene expression in the absence of oxygen stress, were found to be relatively abundant. Some TaDof genes harbored MBS (MYB binding site, which is involved in drought-inducibility), indicating that the expression of these TaDof genes can be influenced by drought. And TaDof gene family members may be involved in defensive damage recovery response and temperature change response due to the presence of WUN-motif, TC-rich repeats, and LTR elements.

Additionally, CCGTCC-box, CAT-box and O2-site accounted for 33.6, 25.1 and $21.7 \%$ of the total number of development related elements, respectively. These elements are involved in the expression and activation of meristematic tissues and the regulation of gliadin metabolism. The promoter-related elements TATA-box and CAAT-box had the largest number of copies per gene, with an average of 13.96 and 15.47, respectively. Except for the promoter of TaDof 86 that had no TATA-box, the other members of the wheat TaDof protein family all contained these two types of cis-acting elements related to transcriptional regulation.

In addition, we also counted the number of each cisacting element present in the subfamily. Interestingly, 


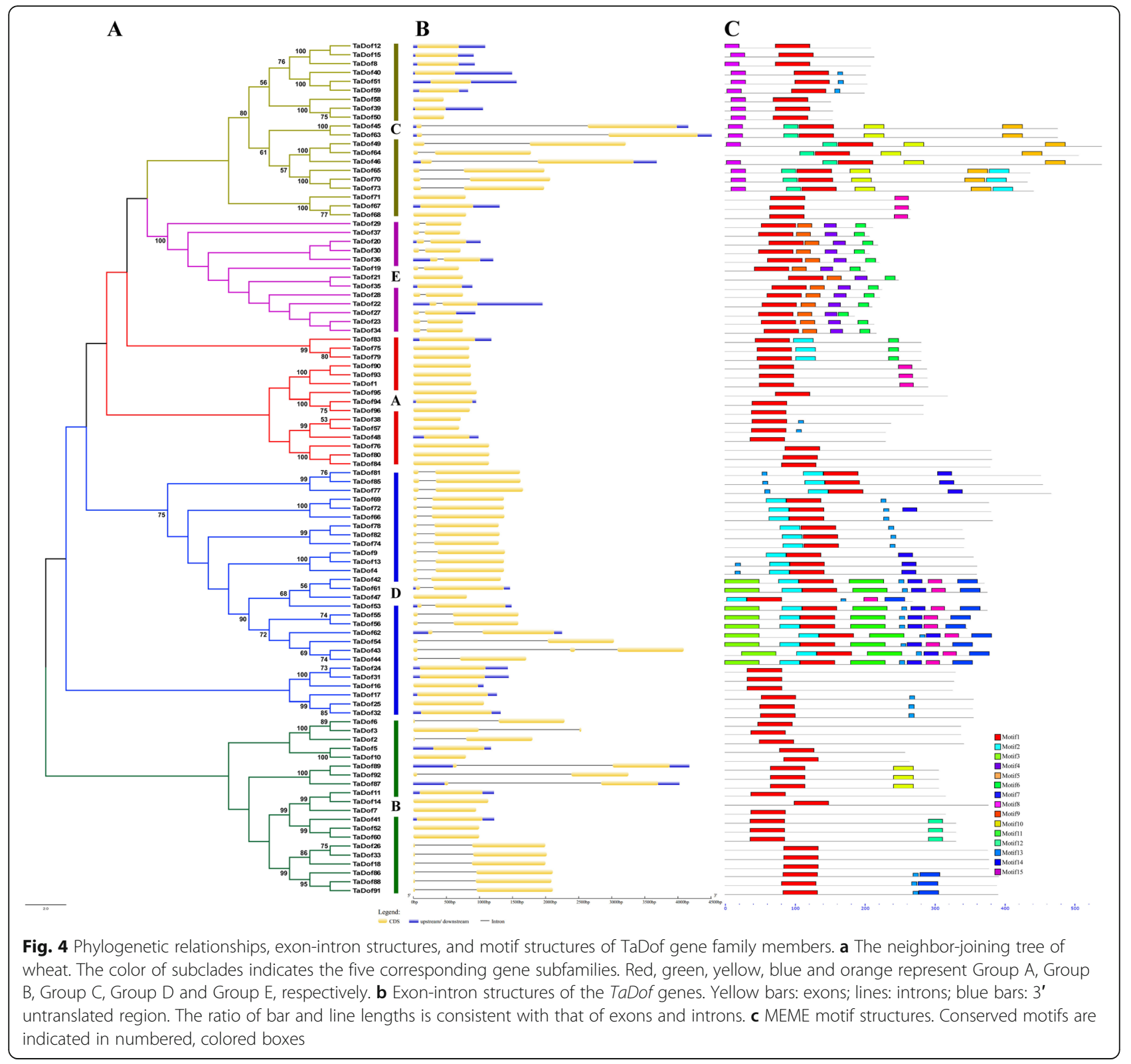

the results showed that the subfamily has a clear preference for the cis-acting elements contained in the seven major classes of cis-acting elements. For example, for light responsive elements, subfamily A had a relatively large number of Abox element, subfamily D had many Box-4 and TCCC-motif elements. Among the development related elements, subfamily A had the largest number of CCGTCC-box, while subfamily D had $59.3 \%$ of the total number of O2-site. For hormone response elements, subfamily A had the most TGACG-motif and CGTCA-motif elements. The number of AREs in the environmental stress-related elements in subfamily D accounted for $51.5 \%$ of the total. Particularly, TATA-box and CAAT-box of promoter binding elements were extremely abundant in subfamily D (Table. S5).

\section{Expression of TaDof genes in different organs and developmental stages}

Analysis of RNA-seq data of different organs at different developmental stages found that TaDof genes showed different expression patterns in different organs and developmental stages (Fig. 6a and Table S6). In general, the 96 TaDof genes could be divided into five groups with distinct expression patterns (Cluster I-V). The seven genes in Cluster I with exhibited a high expression level in leaf, stem and spike, especially at the early developmental stages. However, the expression of some TaDof genes, such as TaDof43, TaDof 38 , TaDof6 and TaDof54, was either very low or undetectable in certain developmental stages. Cluster II contained 17 TaDof 
Table 1 Functional divergence between subfamilies of the TaDof gene family

\begin{tabular}{|c|c|c|c|c|c|c|}
\hline \multirow[t]{2}{*}{ Group1 } & \multirow[t]{2}{*}{ Group2 } & \multicolumn{3}{|l|}{ Type I } & \multicolumn{2}{|l|}{ Type II } \\
\hline & & $\theta_{1} \pm$ s.e. & LRT & Sites with $Q k>0.8$ & $\theta_{\|} \pm$s.e. & Sites with Qk > 0.8 \\
\hline A & B & $0.007 \pm 0.022$ & 0 & None & $-0.044 \pm 0.128$ & $30 A$ \\
\hline A & $C$ & $0.034 \pm 0.022$ & 0 & None & $-0.157 \pm 0.157$ & None \\
\hline A & $D$ & $0.224 \pm 0.090$ & 0.596 & None & $-0.012 \pm 0.129$ & None \\
\hline A & E & $0.418 \pm 0.218$ & 0.786 & None & $-0.007 \pm 0.116$ & $52 \mathrm{~K}, 66 \mathrm{M}, 94 \mathrm{G}, 105 \mathrm{D}$ \\
\hline D & B & $0.177 \pm 0.022$ & 0 & None & $-0.048 \pm 0.129$ & $71 Y$ \\
\hline D & C & $0.022 \pm 0.022$ & 0 & None & $-0.129 \pm 0.163$ & None \\
\hline D & E & $0.272 \pm 0.095$ & 3.989 & $30 \mathrm{~A}$ & $0.053 \pm 0.106$ & 30A, 32A, 33E, 47E $52 \mathrm{~K}, 55 \mathrm{~N}, 66 \mathrm{M}, 94 \mathrm{G}$ \\
\hline B & C & $-0.053 \pm 0.022$ & 0 & None & $-0.094 \pm 0.164$ & $71 Y$ \\
\hline B & E & $0.416 \pm 0.125$ & 1.895 & None & $0.037 \pm 0.118$ & $32 \mathrm{~A}, 45 \mathrm{~K}, 52 \mathrm{~K}, 55 \mathrm{~N}, 66 \mathrm{M}, 71 \mathrm{Y}, 75 \mathrm{~A}, 94 \mathrm{G}$ \\
\hline C & E & $0.310 \pm 0.197$ & 1.115 & None & $-0.082 \pm 0.151$ & $47 \mathrm{E}$ \\
\hline
\end{tabular}

Note: $\theta_{1}$ and $\theta_{\| 1}$ respectively refer to the coefficients of Type-I and Type- II functional divergence between two groups; LRT, Likelihood Ratio Test; Qk, posterior probability. All sites were locatedon the sequence of TaDof6 according to the results of multiple sequence alignment. The bolted amino acid sites are both Type-I and Type- II functional divergence site

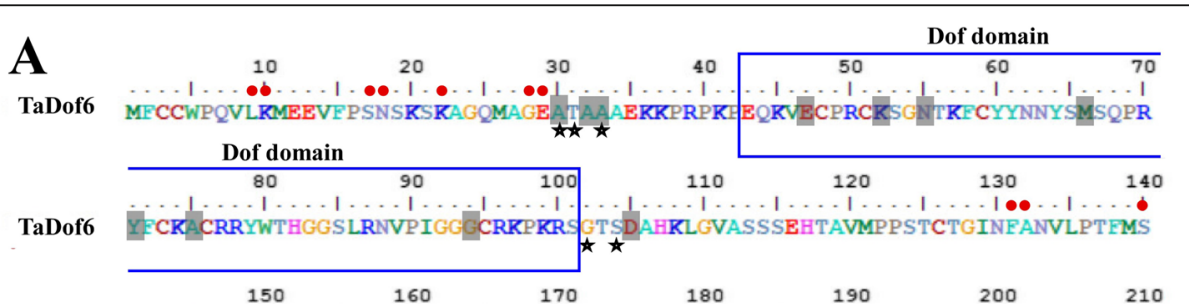

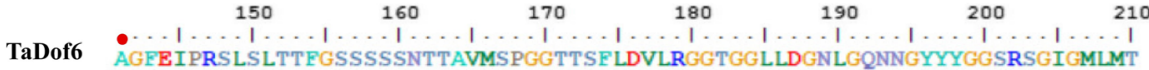

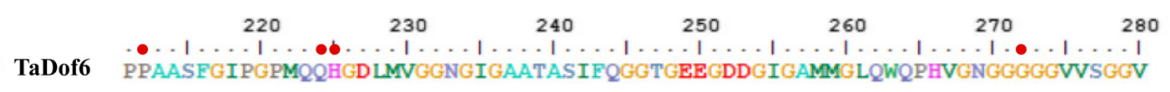

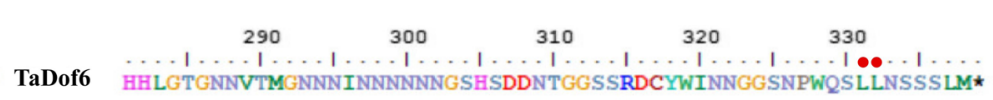

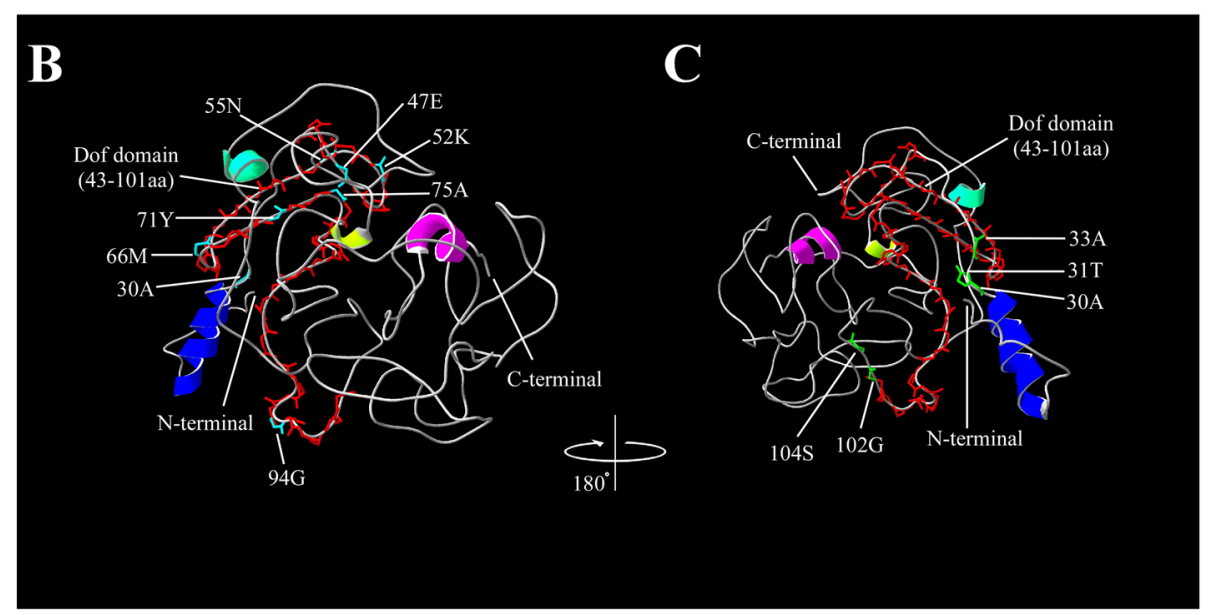

Fig. 5 Protein sequences and model building of the 3D structure of TaDof6. a Protein sequence of TaDof6. The critical amino acid sites of functional divergence, positive selection and coevolution are labeled respectively with gray shadows, black pentagrams and red circles, respectively. $\mathbf{b}$ Schematic diagram of 3D structure of TaDof6. The precise positions of seven critical functional divergence sites were colored in light blue. c Schematic diagram of 3D structure of TaDof6 obtained by (b) rotating 180 degrees anti-clockwise. Five significant positive selection sites are colored in green 
Table 2 Tests for positive selection among TaDofs gene family using site-specific models

\begin{tabular}{|c|c|c|c|c|c|}
\hline Models & $\mathrm{np}$ & Estimates of parameters ${ }^{a}$ & $\operatorname{lnL}$ & $2 \Delta \operatorname{lnL}$ & Positively selected sites ${ }^{b}$ \\
\hline $\begin{array}{l}\text { M0 } \\
\text { (one- } \\
\text { ratio) }\end{array}$ & 191 & $\omega=0.05384$ & - & 883.03 (M3 vs M0) & Not allowed \\
\hline $\begin{array}{l}\text { M3 } \\
\text { (discrete) }\end{array}$ & 195 & $\begin{array}{l}\mathrm{p} 0=0.43174, \mathrm{p} 1=0.26150, \mathrm{p} 2=0.30676 \omega 1= \\
0.00039 \\
\omega 2=0.03024 \\
\omega 3=0.23081\end{array}$ & - & & None \\
\hline $\begin{array}{l}\text { M7 } \\
\text { (beta) }\end{array}$ & 192 & $p=0.19976, q=2.34994$ & - & 2036.983 (M8 va M7) & Not allowed \\
\hline $\begin{array}{l}\text { M8 } \\
\text { (beta \& } \\
\omega \text { ) }\end{array}$ & 194 & $\begin{array}{l}p 0=0.99999, p=0.82856, q=1.22843(p 1= \\
0.00001), \omega=2.55223\end{array}$ & - & & $\begin{array}{l}30 \mathrm{~A}^{* *}, 31 \mathrm{~T}^{* *}, 33 \mathrm{~A}^{* *}, 45 \mathrm{~K}, 67 \mathrm{~S}, 82 \mathrm{H}, 85 \mathrm{~S}, 102 \mathrm{G}^{*} \\
103 \mathrm{~T}, 104 \mathrm{~S}^{* *}, 105 \mathrm{D}\end{array}$ \\
\hline
\end{tabular}

genes that were significantly expressed in all stages of the stem growth, and generally with a low expression in root and high expression in seeds at two and 14 days post anthesis (DPA). In Cluster III, 27 TaDof genes were significantly expressed in spikes or grains, but some genes, such as TaDof55, were not expressed at different growth stages in five organs. In Cluster IV, 22 genes were preferentially expressed in root and their expression levels were relatively low in both leaves and seeds. Cluster V included 23 TaDof genes that were significantly expressed at certain periods in all organs, such as TaDof40, TaDof45 and TaDof59 which were expressed at the late stages of grain development.

It is worth mentioning that most homologous genes had very similar expression patterns during growth and development (Fig. 6a and Fig. 8). Further analysis found that some TaDof genes which clustered in the same subfamily of the phylogenetic tree (Fig. 3) generally also had similar expression patterns. For example, except for TaDof30, all other members of Group E which clustered in Cluster II were significantly expressed in both stems and seeds. However, some other TaDof members, even the homologous genes with highly conserved amino acid sequences in the same subfamily showed distinct expression patterns. For example, 20 TaDof genes in Group C displayed four expression patterns (Cluster I/III/VI/V), of which the expression of two pairs of homologous genes TaDof45/TaDof63 and TaDof51/TaDof59 was distinct. Both TaDof45 and TaDof59 were clustered in Cluster $\mathrm{V}$ and showed higher expression in the spike and grain, while TaDof63 and TaDof51 were clustered in Cluster III and had a peak expression in both spike and root. In particular, the homologous genes TaDof2, TaDof3, and TaDof6 clustered in a small branch of the phylogenetic tree showed a high expression in the endosperm, but the corresponding expression values detected in the qRT-PCR experiment were particularly low in other tissues (Fig. S4).

\section{Expression profiling of TaDof genes in response to various abiotic stresses}

The publicly available RNA-seq data of wheat leaves under polyethylene glycol (PEG)-simulated drought and heat stresses were used to show the expression profile of the TaDof genes (Table S6 and Fig. 6b). Since 10 TaDof genes lacked RNA-Seq atlas data, only 86 genes were analyzed, which were divided into four distinct expression patterns (Cluster A-D in Fig. 6b). Cluster A could be further divided into cluster A1 and cluster A2, and contained 43 genes (50\%). Cluster A1 containing 35 genes was significantly upregulated under heat treatment, and cluster A2 containing 8 genes showed obvious upregulation after heat and PEG treatments. Cluster B had 30 genes $(34.9 \%)$, and more than half of them were upregulated under PEG stress, but all of them were downregulated under heat stress. Cluster $C$ included seven TaDof genes whose expression was downregulated under heat stress, but upregulated under PEG treatment, particularly at $12 \mathrm{~h}$. Six TaDof genes in Cluster D were generally downregulated at the early stages of seedling growth when exposed to PEG stress, but were upregulated at $12 \mathrm{~h}$ after PEG treatment.

To further validate the expression profile of TaDof genes in different organs under various abiotic stresses, we selected 17 representative TaDof genes from the five groups for qRT-PCR analysis, and their primer sequences are listed in Table S7. In leaves, 11 genes (TaDof79, TaDof94, TaDof95, TaDof96, TaDof45, TaDof49, TaDof64, TaDof16, TaDof31, TaDof89 and TaDof29) responded to almost all stress treatments evaluated. In particular, three TaDof genes displayed a significantly upregulated expression in response to multiple abiotic stresses, including TaDof 96 under all stress except for heat stress, TaDof 26 under ABA, PEG, and cold treatments, and TaDof35 under $\mathrm{Cr}^{3+}, \mathrm{Cd}^{2+}$, and heat treatments. All TaDof genes except TaDof96 and TaDof26 were significantly downregulated under cold 


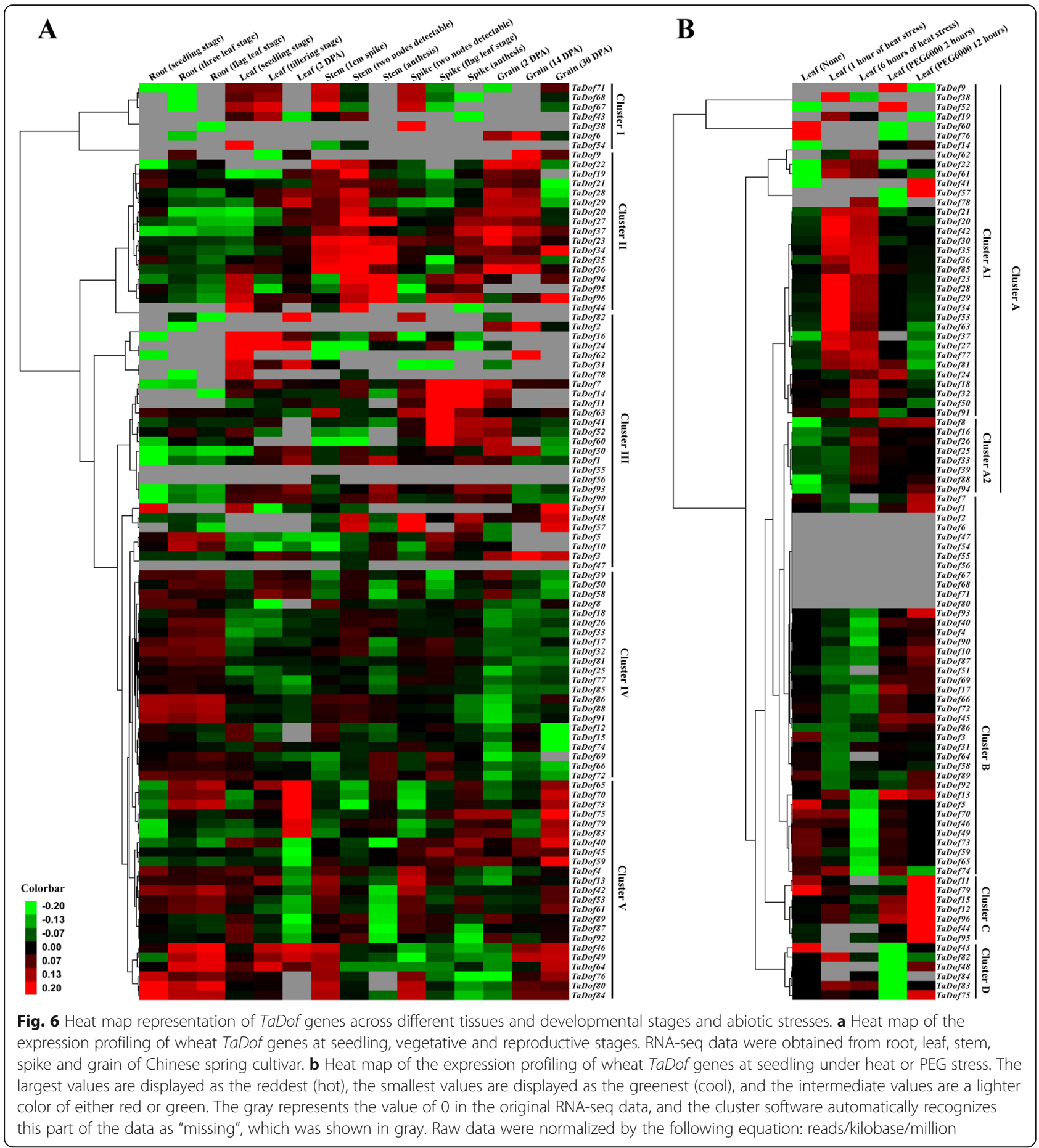

treatment, whereas all genes, except TaDof16, TaDof26 and TaDof 49 , were significantly upregulated under heat treatment. However, both TaDof16 and TaDof26 were significantly upregulated under PEG treatment. These results are generally consistent with the RNA-seq data described above (Table S8). According to the results of RNA-seq datas and qRT-PCR, the expression levels of the most homologous genes in response to abiotic stress were significantly different. For example, TaDof 96 was significantly upregulated under all stress treatments except for heat stress, but the homologous genes (TaDof 94 and TaDof95) were downregulated under most stress treatments (Figs. 6b and 7).

In addition, seven TaDof genes (TaDof79, TaDof95, TaDof96, TaDof49, TaDof64, TaDof16 and TaDof29) showed a sensitive response to hormone stress, in which 


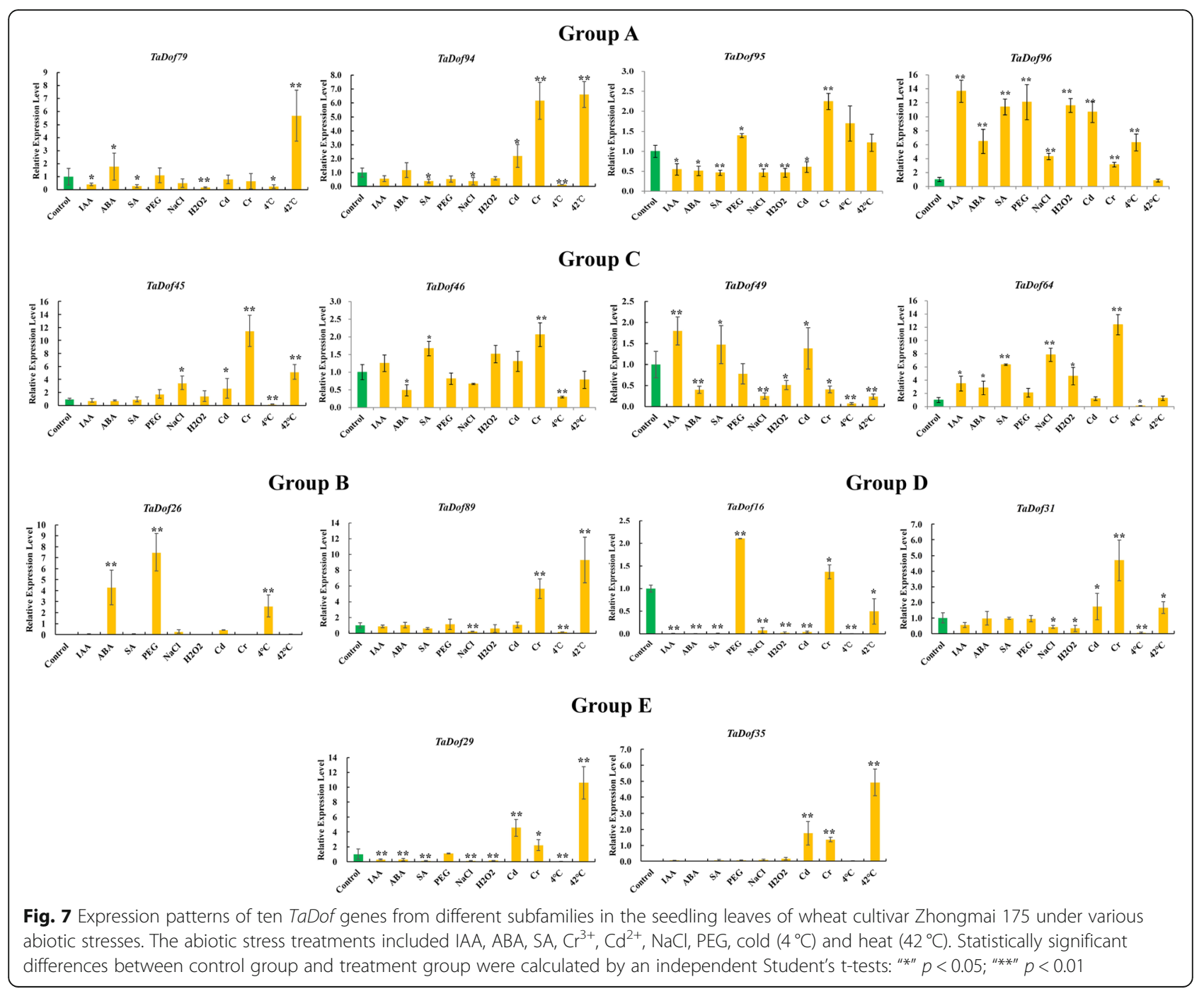

TaDof 96 and TaDof 64 were significantly upregulated under three hormone stresses, TaDof 49 was significantly upregulated under IAA and SA treatment and the other genes were generally downregulated under hormonal stress. When subjected to heavy metal stress, the expression of most TaDof genes was significantly upregulated, especially under $\mathrm{Cr}^{3+}$ stress. Additionally, six TaDof genes (TaDof79, TaDof95, TaDof49, TaDof16, TaDof31, and TaDof29) were significantly downregulated after oxidative stress. Under salt stress, seven TaDof genes (TaDof94, TaDof95, TaDof89, TaDof16, TaDof49, TaDof31 and TaDof29) were downregulated, whereas three TaDof genes (TaDof96, TaDof45, and TaDof64) were significantly upregulated (Fig. 7).

In grains, three highly expressed TaDof genes (TaDof2, TaDof3 and TaDof6) showed up-and-down expression patterns along with grain development, except for TaDof6 under nitrogen treatment. Their expression was highly induced and inhibited by high-nitrogen and low- nitrogen treatment, respectively. The effects of nitrogen stress on the expression of the TaDof3 and TaDof6 genes were more obvious during grain development (Fig. 8a). Under drought stress, three TaDof genes were significantly upregulated in the early developmental stages of the wheat cultivars Zhongmai 175 and Jimai 22 (Fig. 8b).

\section{Discussion}

Molecular characterization and evolution of wheat Dof transcription factors

According to the cluster analysis results of A. thaliana and rice [3,32], the sorghum Dof gene family was divided into six subfamilies (Group A-F), of which Group B had only four AtDof members, namely AtDof4.2, AtDof 4.3, AtDof4.4 and AtDof4.5 [34]. In this study, we used the recently released wheat genome database to identify 96 Dof genes at the genome-wide level (Table S2). The 96 Dof genes were classified into five subfamilies (Group 

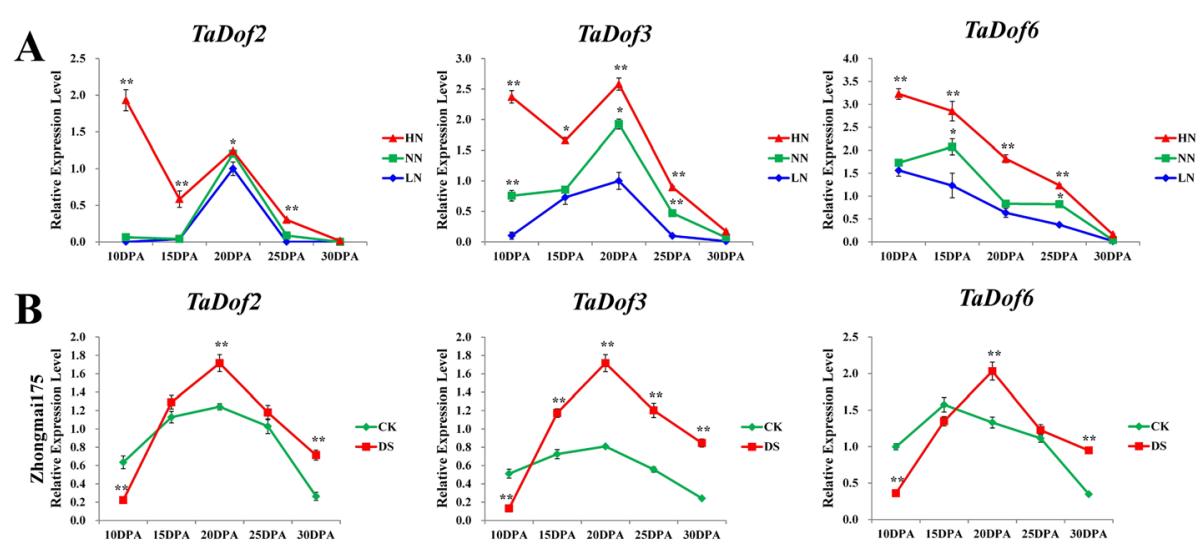

TaDof3

TaDof6
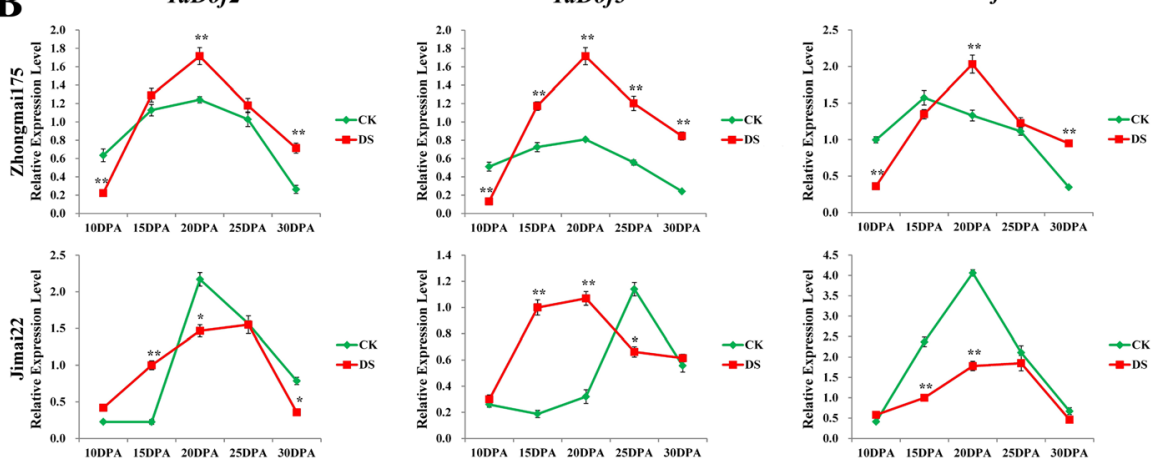

Fig. 8 Expression pattern of wheat TaDof2/3/6 genes. a Expression patterns of TaDof2/3/6 genes during grain development of wheat cultivar Zhongmai 175 and in response to high and low nitrogen treatments. LN (low-nitrogen): without fertilization application after sowing; NN (normal nitrogen): fertilization with $180 \mathrm{~kg} / \mathrm{hm}^{2}$; HN (high-nitrogen): fertilization with $240 \mathrm{~kg} / \mathrm{hm}^{2}$. b Expression patterns of TaDof2/3/6 genes during grain development of wheat cultivars Zhongmai 175 and Jimai 22 and in response to drought stress. CK: the control group with two-irrigation at jointing and anthesis stages; DS: drought stress group without irrigation after sowing. Expression of the wheat Ubiqutin gene was used as a reference gene. Statistically significant differences between control group and treatment group were calculated by an independent Student's ttests: "** $p<0.05 ;{ }^{\prime * * "} p<0.01$

A-E) using the Bayesian method, in which the four AtDof members were categorized into Group A (Fig. 3), slightly different from sorghum Dof gene classification.

The exon-intron organization can be used as supporting evidence to determine the evolutionary relationships among genes or organisms $[48,49]$. In general, the wheat TaDof genes in the same subfamily share similar exon-intron structures, but differences are present in different subfamilies. The distribution of motifs among Dof proteins is indicative of evolutionary relationship as deduced by phylogenetic tree [50]. The results shown in Fig. 4 demonstrated that the sequence structure of motif 1 identified by MEME was consistent with the Dof domain, which may be involved in binding to a particular promoter sequence. Meanwhile, at least one or two conservative motif types and spatial arrangements in TaDofs are present in the same subfamily, but obvious differences occur between different subfamilies, implying certain functional similarities of Dof members within the same subfamily. In addition, the structural conservation of the TaDof genes in subfamilies was consistent with other plants such as Arabidopsis and rice [32], barley [33], sorghum [34] and B. distachyon [35].

Wheat genomes (BBAADD) consist of three related sub-genomes, and they were derived from three different diploid species, respectively [51]. A total of 96 TaDofs transcription factor family members were identified in this study. They were unequally distributed in sub- genomes $\mathrm{A}, \mathrm{B}$, and $\mathrm{D}$, with 34,32 , and 30 members, respectively (Fig. 1). This suggests that there may be a loss of homologous genes during evolution. On the other hand, the retained genes and their distribution on chromosomes provided valuable reference for the polyploidization of wheat chromosomes. It is worth noting that the TaDof gene on chromosome 4A was opposite to the distribution of homologous genes on chromosomes $4 \mathrm{~B}$ and $4 \mathrm{D}$, basically consistent with the recent research [52]. This indicates that the chromosome $4 \mathrm{~A}$ arm had been reversed during the evolution of hexaploid wheat [53]. We also found that the genes located at the lower part of chromosomes 2 and 3 were closely arranged, and their sequences were highly consistent. They meet the definition of tandem duplication, so they are considered as tandem duplication genes formed during long-term evolution. Interestingly, although the sequences of TaDof 47 and TaDof $42-44$ are highly similar, there are too many genes between them to consider as a tandem duplication gene. Moreover, it didn't meet the requirements of segmental duplication gene, so we speculate that it may be caused by the insertion of a short chromosome segmental between TaDof44 and TaDof 47 during the evolution. The functional diversity of proteins is generally resulted from gene duplication events or the formation of new species [54]. According to our results, type-II functional divergence sites were significantly more abundant than type-I functional divergence sites 
(Table 1), demonstrating the important roles of the physical and chemical property changes of amino acids in the diversification among the subfamilies. All seven type-II functional divergence sites (47E, $52 \mathrm{~K}, 55 \mathrm{~N}, 66$ $\mathrm{M}, 71 \mathrm{Y}, 75 \mathrm{~A}$ and $94 \mathrm{G}$ ) are located in the Dof domain (Fig. 5), indicating that they may be the main driving force promoting the variation of the Dof domain and the functional divergence of the wheat Dof family. At the molecular level, the amino acid mutations that increase adaptation to the environment were retained through positive selection [55]. Five sites (30A, $31 \mathrm{~T}, 33 \mathrm{~A}, 102 \mathrm{G}$ and $104 \mathrm{~S}$ ) were found to have been subjected to strong positive selection, and may play important roles in functional divergence. Meanwhile, complex coevolutionary networks play important roles in the stability of protein structure and function during evolutionary process [46]. Nine coevolutionary amino acid sites detected in TaDof family members (Table S3) may play roles in maintaining the spatial structure of Dof proteins. These coevolutionary sites may not only perform an important function, but also play a key role in the evolution of the wheat Dof gene family. Therefore, these special amino acid sites might provide useful information for further deciphering the functional attributes of TaDof genes.

\section{Potential roles of TaDof genes in tissue differentiation and organ development}

Phylogenetic and expression analyses can provide important clues about the potential functions of wheat Dof genes. As reported in other plants [36, 56, 57], the TaDof genes showed a specific and preferential expression in different organs and developmental stages (Fig. 6a), implying their involvement in plant growth and development. Phylogenetic analysis revealed that eight TaDof genes (TaDof45/46/49/63/64/65/70/73) showed a high sequence similarity to the five $A$. thaliana Cycling DOF Factors (CDF1-5) in the Group C subfamily, and they were expressed in the developing grains and stem/spike at the flowering stage (Fig. 3). CDF1 represses the transcription of Constans $(C O)$ and thereby represses flowering in $A$. thaliana [58]. Thus, it is likely that these TaDof genes also play roles in spike and seed development.

The homologous TaDof2, TaDof3 and TaDof6 genes were highly expressed in the early and middle stages of grain development (Fig. 6a). They were closely clustered with $R P B F$ (OsDof7) in the phylogenetic tree (Fig. 3), suggesting their functional similarity. The previous study showed that $R P B F$ gene, predominantly expressed in the maturing endosperm and coordinately expressed with seed storage protein genes, also participated in the quantitative regulation of genes expressed in the endosperm through cooperating with RISBZ1 [19]. Wheat WPBF can trans-activate the native alpha-gliadin gene promoter via interaction with the 5'-TGTAAAG-3' motif
[28]. As PBF homologues, the proteins encoded by the TaDof2, TaDof3 and TaDof6 genes activate wheat prolamin gene expression during seed development [26, 27]. Furthermore, the promoter activities of WPBF were observed in the vascular system and the seeds of transgenic A. thaliana, suggesting that WPBF functions not only in seed development, but also during other plant growth and developmental stages [28]. We found that some development-related elements present in TaDof2/3/6 are involved in seed-specific regulation (O2-site, RYelement) and meristem expression (CAT-box) or specific activation (CCGTCC-box) (Table S4), which could play important roles in regulating plant growth and seed development.

It is well established that stems contained abundant vascular tissue. The TaDof genes in Group E showed predominantly higher transcript levels in stem (Fig. 6), suggesting they may play a role in vascular tissue development. A. thaliana Dof factor OBP1 (OBF-binding factor-1) is implicated in a more general control of cell division, and plays an important role in cell cycle reentry, acting as a transcriptional regulator of key cell cycle genes [59]. Promoter activity analysis suggested that AtDof5.8 may function in the primary processes of leaf vasculature formation. AtDof5.8 promoter sequences also contain cis-elements for the stage-specific expression during vascular development [60]. In this study, we detected a large number of the meristem-specific activation-related element CCGTCC-box in the TaDof gene promoter regions of Group E, and each gene contained more than 2.3 copies (Table S5), indicating that these TaDof genes may also function in various vascular development processes.

\section{Expression and functions of TaDof genes in response to abiotic stresses}

Dof transcription factors are involved in various abiotic stress responses through the regulation of multiple metabolic pathways. Dof proteins are implicated in responses to plant hormones, such as auxins [61] and gibberellins [8]. When a stressor causes an increase in auxin, the plant activates $\mathrm{ABA}$ and other pathways, ultimately promoting the expression of stress-defense genes [62, 63]. The StDof genes in potato showed either ABA-dependent or ABAindependent expression pattern [64]. We detected a numerous phytohormone regulation-related elements in the promoter region of the $96 \mathrm{TaDof}$ family members, such as the TCA-element, GARE-motif, TGA-element, ABRE and CGTCA-motif (Table S4). qRT-PCR analysis also revealed that the expression of TaDof genes can be induced by IAA, ABA and SA (Fig. 7), indicating that these cis-elements related to the phytohormone response play important roles in plant hormone pathways. 
Reactive oxygen species (ROS) in plants remain at a relatively stable level under normal physiological conditions. However, heavy metal stress will cause an imbalance in the production and clearance of $\mathrm{H}_{2} \mathrm{O}_{2}$, leading to oxidative damage to plant cells due to excessive accumulation of ROS $[65,66]$. We found that eight TaDof genes (TaDof95, TaDof96, TaDof46, TaDof49, TaDof64, TaDof16, TaDof31 and TaDof29) were significantly upregulated under heavy metal stress (Fig. 7). We speculate that TaDof genes can act as important regulators in the dynamic regulation of ROS clearance pathways.

The AtDof gene CDF3 is highly induced by drought, extreme environment as well as ABA. Studies showed that $c d f 3-1$, a $C D F 3$ T-DNA insertion mutant, is much more sensitive to drought and low temperature stresses, whereas $C D F 3$ overexpression promotes the tolerance to drought, cold and osmotic stress [12, 17]. Five homologues of $A$. thaliana CDFs (SlCDF1-5) were reported to be differentially induced in response to osmotic, salt, heat, and low-temperature stresses [25]. Most of the Dof genes in Chinese cabbage were also quickly upregulated by salt, drought, heat, and cold stress treatments [56]. Furthermore, the Dof14-15 and Dof1 genes in wheat were significantly upregulated under drought and salt stresses [27]. In this study, most TaDof genes were upregulated under heat stress, and the RNA-Seq data also revealed that the majority of the TaDof genes were responsive to heat and PEG stresses (Fig. 6). In particular, the TaDof16, TaDof 26 and TaDof 96 genes were upregulated two-, seven- and twelve fold after PEG stress, respectively (Fig. 7). A large number of elements related to environmental stress were also present in the promoter region of TaDof genes such as LTR and MBS, and each member had more than 1.2 copies of ARE, GC-motif and WUN-motif (Table S4), which may play an important role in responding to various abiotic stresses.

Introduction of the maize $Z m D o f 1$ gene into rice can increase nitrogen assimilation and enhance plant growth under low-nitrogen conditions [67]. Dof1 and GS work together to regulate the nitrogen metabolism pathway in plants, and to enhance nitrogen assimilation in transgenic tobacco plants grown under low-nitrogen conditions [68]. The TaDof2, TaDof3 and TaDof6 genes were highly expressed during grain development under drought stress, which improved the synthesis of storage proteins and gluten quality [30]. Consistent with previous studies, in this study, the expression of the TaDof2, TaDof 3 and TaDof6 genes was upregulated under drought and nitrogen stresses (Fig. 8). These results suggest the potential roles of TaDof genes in the response to abiotic stresses.

\section{Conclusions}

A total of $96 \mathrm{TaD}$ of transcription factor genes were identified from the wheat genome database, which were classified into five subfamilies (Group A-E). The members of the TaDof family almost had no introns and all contained a conserved Dof domain. Type-II function divergence was identified as the main reason for the functional diversification of TaDof transcription factors. The Dof gene family underwent different degrees of positive selection pressure in the evolutionary process. The nine coevolutionary sites were identified, which may play important roles in maintaining the structural and functional stability of TaDof proteins. Depending on the type and number of cis-acting elements, the TaDof genes may be regulated by a variety of hormones and environmental factors. RNA-seq data analysis found that TaDof genes showed multiple expression patterns, with obvious expression specificity and preference in different organs and developmental stages, suggesting their potential roles in tissue differentiation and organ development. qRT-PCR analysis further showed that some TaDof genes were significantly upregulated in response to single and multiple abiotic stressors, suggesting that they are involved in stress resistance. Our study provides valuable information for further understanding the molecular evolutionary mechanism and functional traits of the plant Dof gene family.

\section{Methods \\ Identification of Dof transcription factors in wheat}

The amino acid sequences of 36 and 30 Dof transcription factors of Arabidopsis thaliana and Oryza sativa were firstly downloaded from the plant transcription factor database (PlantTFDB v4.0, http://planttfdb.cbi.pku. edu.cn/) $[32,69]$. Then the obtained Dofs protein sequences of rice and Arabidopsis were used for BLASTP operation (E-value $\leq 1 \mathrm{e}-5$ ) to retrieve possible TaDofs in wheat from the published common wheat genome database (IWGSC RefSeq v1.0) in Ensemble Plants (http:// plants.ensembl.org/). The SMART (http://smart.emblheidelberg.de/) [70] and Pfam (http://pfam.xfam.org/) [71] websites were used to further verify if the identified proteins had a conserved Dof domain.

\section{Chromosome location and gene duplication analysis of TaDof genes}

The chromosome location of TaDof genes was obtained from the IWGSC database and visualized by the MapInspect software. The duplication gene pairs in the Dof gene family were identified based on the criteria of the previous studies [52, 72]. For segmental duplication analysis, we used a pair of non-homologous but similar genes as the center to take 10 genes from the upstream and downstream respectively to compare the similarity of these genes. Based on the similarity and arrangement of the genes on the chromosomes near the two genes, it was determined whether the two genes were segment 
duplicate genes. The tandem duplicate analysis of TaDof genes was performed according to the method described by Yin et al. [73].

\section{Subcellular localization of TaDof proteins}

The prediction and experimental verification of subcellular localization of TaDofs were performed according to the website and experimental method described by Liu et al. [74].

\section{Phylogenetic analysis}

Multiple sequence alignment of the amino acid sequences of Dof proteins were performed based on MUSCLE program (http://www.ebi.ac.uk/Tools/msa/ muscle/) $[75,76]$. Subsequently, the Bayesian and N-J phylogenetic trees were constructed based on the method by Han et al. [77]. Furthermore, maximum likelihood and minimal evolution methods were applied for the tree construction to validate the results of the $\mathrm{N}-\mathrm{J}$ method in MEGA5.0.

\section{Sequence characteristics analysis}

The PlantCARE (http://bioinformatics.psb.ugent.be/ webtools/plantcare/html/) was used to analyze cis-acting elements in the $1500 \mathrm{bp}$ promoter region upstream of the TaDofs [47]. The exon-intron structures map of TaDofs were detected by comparing the coding sequences (CDS) and genomic sequences in Gene Structure Display Server v2.0 website (GSDS, http://gsds.cbi. pku.edu.cn/) [78]. Conserved motifs of the TaDof protein sequences were detected by MEME website (http:// meme-suite.org/tools/meme), the maximum number of motifs was set to 15 and the remaining operating parameters were unchanged $[79,80]$. Theoretical $\mathrm{pI} / \mathrm{MW}$ of TaDofs was calculated by the Compute pI/MW tool (http://web.expasy.org/compute_pi/).

\section{Molecular evolution analysis of the TaDofs gene family} DIVERGE 3.0 software package was used to detect the presence of type I and type II functional divergence sites in different subfamilies, and the critical value of posterior probability $(\mathrm{Qk})$ was set to 0.8 [43]. The positive selection analysis of the Dof protein family was performed using site model in the PAML4.4 software package [45, 81, 82]. Coevolution sites between amino acids were identified using Coevolution Analysis using Protein Sequences (CAPS) in PERL software [46, 83].

\section{Three-dimensional (3D) structure analysis of TaDof proteins}

The 3D structures of TaDof proteins were constructed using PHYRE2 website (http://www.sbg.bio.ic.ac.uk/ phyre2/html/page.cgi?id=index) [84], and visualized by Pymol software (http://pymol.org/).

\section{TaDof gene expression analysis by RNA-seq data}

The RNA-seq data of the TaDof genes were downloaded from the expVIP website (http://www.wheat-expression. $\mathrm{com} /$ ) [85], and cluster analysis was performed by Cluster 3.0 and TreeView software.

\section{Plant material and abiotic stress treatments}

The Chinese wheat cultivar Zhongmai 175 seedlings were cultivated into two leaves and one heart stage according to the culture conditions of Han et al. [77]. Then seedlings were treated with the following conditions: heavy metal stress with $300 \mu \mathrm{M} \mathrm{CrCl}_{3}$ and $\mathrm{CdCl}_{2}$, drought stress with 20\% (w/v) PEG 6000, salinity stress with $200 \mathrm{mM} \mathrm{NaCl}$, oxidative stress with $15 \mathrm{mM} \mathrm{H}_{2} \mathrm{O}_{2}$, hormone stress with $100 \mu \mathrm{M}$ ABA (abscisic acid) and SA (salicylic acid), and $10 \mu \mathrm{M}$ IAA (indole-3-acetic acid), cold stress under $4{ }^{\circ} \mathrm{C}$ ) and heat stress under $42^{\circ} \mathrm{C}$. The samples from heat stress were collected at $2 \mathrm{~h}$, and other treated seedlings were harvested at $12 \mathrm{~h}$. Three biological replicates were set up for each sample, which were quickly frozen in liquid nitrogen and stored at $-80^{\circ} \mathrm{C}$ refrigerator.

Meanwhile, two elite Chinese wheat cultivars Zhongmai 175 and Jimai 22 were planted at the experimental station of China Agricultural University, Wuqiao, Hebei Province $\left(116^{\circ} 37^{\prime} 23^{\prime \prime} \mathrm{E}\right.$ and $\left.37^{\circ} 16^{\prime} 02^{\prime \prime} \mathrm{N}\right)$ during the 2016-2018 wheat growing season. Field experiments included two-irrigation at jointing and anthesis stages for control group (CK), no-irrigation after sowing for drought stress treatment (DS), low-nitrogen (LN) without fertilization application after sowing, normal nitrogen $(\mathrm{NN})$ fertilization with $180 \mathrm{~kg} / \mathrm{hm}^{2}$ and highnitrogen $(\mathrm{HN})$ fertilization with $240 \mathrm{~kg} / \mathrm{hm}^{2}$. Each treatment contained three biological replicates and each plot had $20 \mathrm{~m}^{2}$. Plants were marked after flowering, and grain samples from five developmental stages of 10, 15, 20, 25 and 30 days post-anthesis (DPA) were harvested. All samples were quickly collected and immediately frozen in liquid nitrogen, and then stored at $-80^{\circ} \mathrm{C}$ prior to analysis.

\section{qRT-PCR analysis}

Total RNA extraction, primer design, qRT-PCR procedures and data analysis were performed according to Cao et al. [86], and three biological replicates were used for each sample. Ubiquitin gene was used as the reference gene. The expression levels of the target genes were determined according to the expression changes relative to the reference gene. At the beginning of the experiment, the relative stability of the reference gene was tested by using various organs and wheat materials treated with different abiotic stress treatments. 


\section{Supplementary information}

Supplementary information accompanies this paper at https://doi.org/10. 1186/s12864-020-6691-0.

Additional file 1: Figure S1. The Neighbour-Joining tree of Dof transcription factor gene family from Triticum aestivum L., Arabidopsis thaliana and Oryza sativa L.

Additional file 2: Figure S2. The phylogenetic tree of Dof transcription factor gene family from Triticum aestivum L. (A) Minimun Evolution Tree. (B) Maximun Likelihood tree.

Additional file 3: Figure S3. Motifs of TaDof proteins.

Additional file 4: Figure S4. Expression profiling of TaDof2, TaDof3 and TaDof6 genes in seven wheat tissues and organs.

Additional file 5: Table S1. The nomenclature, characteristics of Dof genes and their deduced proteins in Arabidopsis thaliana and Oryza sativa subsp. japonica. The data (accession numbers and amino acid sequences) of Dof transcription factor for Arabidopsis and rice are obtained from the Plant Transcription Factor Database (http://planttfdb. cbi.pku.edu.cn/family.php?sp=Ath\&fam=Dof; http://planttfdb.cbi.pku.edu. cn/family.php?sp=Osj\&fam=Dof). Table S2. The nomenclature, characteristics of Dof genes and their deduced proteins in Triticum aestivum $L$. The accession numbers and sequences (genomic, amino acid, and CDS sequences) of the 96 wheat Dof genes were obtained from Triticum aestivum genome data (IWGSC) in Ensemble Plants (http://plants. ensembl.org/Triticum_aestivum/Info/Index). Table S3. Coevolution sites in TaDofs. Table S4. Cis-element analysis of $1500 \mathrm{bp}$ nucleotide sequences data upstream of the translation initiation codon of TaDof genes. Table S5. The number of each cis-acting element contained in the subfamily. Table S6. The RNA-Seq atlas data of the TaDof genes. Table S7. Primers used for qRT-PCR of Dof genes in wheat. Table S8. The relative expression values in GRT-PCR and RNA-Seq atlas data of TaDofs under abiotic stresses. (A) The relative expression values of TaDofs in qRT-PCR under abiotic stresses. (B) The RNA-Seq atlas data of TaDofs under abiotic stresses.

\section{Abbreviations}

ABA: Auxin, abscisic acid; CDF: Cycling DOF Factors; CDS: Coding sequence; CK: Control group; Dof: DNA binding with one finger; DPA: Days post anthesis; DS: Drought stress treatment; GFP: Green fluorescent protein; GSDS: Gene structure display server; HN: High-nitrogen; IAA: Indole-3-acetic acid; IWGSC: International Wheat Genome Sequencing Consortium; LN: Lownitrogen; LRT: Likelihood ratio test; MBS: MYB binding site; MCMC: Markov Chain Monte Carlo; MEJA: Methyl jasmonic acid; NJ: Neighbor-joining; NN: Normal nitrogen; OBP: OBF-binding factor; PBF: Prolamin-box binding factor; PEG: Polyethylene glycol; PlantTFDB: Plant Transcription Factor Database; RPBF: Rice PBF; SA: Salicylic acid; 3D: Three-dimensional; TFs: Transcription factors

\section{Acknowledgements}

The English in this document has been checked by professional editor, native speakers of English. For a certificate, please see: www.editorbar.com/ order/cert/ LE201906130135.

\section{Authors' contributions}

$Y L$ and NN L carried out the bioinformatic analysis, experimental treatments and QRT-PCR. XD, DM L, MF L and DD C participated in the study and helped to draft the manuscript. YM $Y$ and $Y K$ H designed the study and provide guidance on the whole study. All authors read and approved the final manuscript.

\section{Funding}

This research was financially supported by grants from National Key R \& D Program of China (2016YFD0100502) and the National Natural Science Foundation of China $(31771773,31471485)$. The funders had no role in the study process including preparation of the manuscript.

\section{Availability of data and materials}

The 96 wheat Dof genes data and their accession numbers listed in Table S2 were derived from Triticum aestivum genome data (IWGSC) in Ensemble
Plants (http://plants.ensembl.org/Triticum_aestivum/Info/Index), and all accession numbers allow access to public data. The wheat genome sequencing data access URL in Ensemble Plants is ftp://ftp.ensemblgenomes. org/pub/release-46/plants/fasta/triticum_aestivum/dna/. The data (accession numbers and amino acid sequences) of Dof transcription factor for Arabidopsis and rice are obtained from the Plant Transcription Factor Database (http://planttfdb.cbi.pku.edu.cn/family.php?sp=Ath\&fam=Dof; http://planttfdb.cbi.pku.edu.cn/family.php?sp=Osj\&fam=Dof). The RNA-seq data of the 96 wheat Dof genes with specific accession numbers from Ensemble Plants (http://plants.ensembl.org/Triticum_aestivum/Info/Index) are available on the expVIP website (http://www.wheat-expression.com/), and the transcriptome data of hexaploid wheat tissues, cultivars and stress conditions access URL in expVIP website is https://opendata.earlham.ac.uk/wheat/ under_license/toronto/Ramirez-Gonzalez_etal_2018-06025-TranscriptomeLandscape/expvip/RefSeq_1.1/ByTranscript/. All data generated or analyzed during this study are included in this published article and its supplementary information files.

\section{Ethics approval and consent to participate}

Not applicable

\section{Consent for publication}

Not applicable.

\section{Competing interests}

The authors declare that they have no competing interests.

\section{Author details}

'College of Life Science, Capital Normal University, Xisanhuan Beilu No. 105, 100048 Beijing, People's Republic of China. ${ }^{2}$ Hubei Collaborative Innovation Center for Grain Industry (HCICGI), Yangtze University, Jingzhou 434025, China.

Received: 26 July 2019 Accepted: 24 March 2020

Published online: 03 April 2020

\section{References}

1. Gupta S, Malviya N, Kushwaha H, Nasim J, Bisht NC, Singh VK, Yadav D. Insights into structural and functional diversity of Dof (DNA binding with one finger) transcription factor. Planta. 2015;241:549-62.

2. Yanagisawa S, Schmidt RJ. Diversity and similarity among recognition sequences of Dof transcription factors. Plant J. 1999;17:209-14.

3. Yanagisawa S. The Dof family of plant transcription factors. Trends Plant Sci. 2002;7:555-60.

4. Umemura Y, Ishiduka T, Yamamoto R, Esaka M. The Dof domain, a zinc finger DNA-binding domain conserved only in higher plants, truly functions as a Cys2/Cys2 Zn finger domain. Plant J. 2004;37:741-9.

5. Vicente-Carbajosa J, Moose SP, Parsons RL, Schmidt RJ. A maize zinc-finger protein binds the prolamin box in zein gene promoters and interacts with the basic leucine zipper transcriptional activator opaque2. Proc Natl Acad Sci U S A. 1997;94:7685-90.

6. Kang HG, Singh K. Characterization of salicylic acid-responsive Arabidopsis Dof domain proteins: overexpression of OBP3 leads to growth defects. Plant J. 2000;21:329-39.

7. Zhang B, Chen W, Foley RC, Büttner M, Singh KB. Interactions between distinct types of DNA binding proteins enhance binding to ocs element promoter sequences. Plant Cell. 1996;7:2241-52.

8. Washio K. Functional dissections between GAMYB and Dof transcription factors suggest a role for protein-protein associations in the gibberellinmediated expression of the RAmy1A gene in the rice aleurone. Plant Physiol. 2003;133:850-63.

9. Diaz I, Vicente-Carbajosa J, Abraham Z, Martinez M, Moneda II, Carbonero P. The GAMYB protein from barley interacts with the DOF transcription factor BPBF and activates endosperm-specific genes during seed development. Plant J. 2002:29:453-64.

10. Rueda-Romero P, Barrero-Sicilia C, Gomez-Cadenas A, Carbonero P, OnateSanchez L. Arabidopsis thaliana DOF6 negatively affects germination in nonafter-ripened seeds and interacts with TCP14. J Exp Bot. 2012;63:1937-49.

11. Kang HG, Foley RC, Onate-Sanchez L, Lin CGT, Singh KB. Target genes for OBP3, a Dof transcription factor, include novel basic helix-loop-helix domain proteins inducible by salicylic acid. Plant J. 2003;35:362-72. 
12. Renau-Morata B, Molina RV, Carrillo L, Cebolla-Cornejo J, Sánchez-Perales M, Pollmann S, Domínguez-Figueroa J, Corrales AR, Flexas J, Vicente-Carbajosa J, Medina J, Nebauer SG. Ectopic expression of CDF3 genes in tomato enhances biomass production and yield under salinity stress conditions. Front Plant Sci. 2017;8:660.

13. Zou HF, Zhang YQ, Wei W, Chen HW, Song QX, Liu YF, Zhao MY, Wang F, Zhang BC, Lin Q, Zhang WK, Ma B, Zhou YH, Zhang JS, Chen SY. The transcription factor AtDOF4.2 regulates shoot branching and seed coat formation in Arabidopsis. J Biochem. 2013;449:373-88.

14. Guo Y, Qin G, Gu H, Qu LJ. Dof5.6/HCA2, a Dof transcription factor gene, regulates interfascicular cambium formation and vascular tissue development in Arabidopsis. Plant Cell. 2009;21:3518-34.

15. Imaizumi T, Schultz TF, Harmon FG, Ho LA, Kay SA. FKF1 F-box protein mediates cyclic degradation of a repressor of CONSTANS in Arabidopsis. Science. 2005;309:293-7.

16. Fornara F, Panigrahi KC, Gissot L, Sauerbrunn N, Ruhl M, Jarillo JA, Coupland $\mathrm{G}$. Arabidopsis DOF transcription factors act redundantly to reduce CONSTANS expression and are essential for a photoperiodic flowering response. Dev Cell. 2009;17:75-86.

17. Corrales AR, Carrillo L, Lasierra P, Nebauer SG, Dominguez-Figueroa J, Renau-Morata B, Pollmann S, Granell A, Molina RV, Vicente-Carbajosa J, Medina J. Multifaceted role of cycling DOF factor 3 (CDF3) in the regulation of flowering time and abiotic stress responses in Arabidopsis. Plant Cell Environ. 2017;40:748-64.

18. Negi J, Moriwaki K, Konishi M, Yokoyama R, Nakano T, Kusumi K, HashimotoSugimoto M, Schroeder Jl, Nishitani K, Yanagisawa S, Iba K. A Dof transcription factor SCAP1 is essential for the development of functional stomata in Arabidopsis. Curr Biol. 2013;23:479-84

19. Yamamoto MP, Onodera Y, Touno SM, Takaiwa F. Synergism between RPBF Dof and RISBZ1 bZIP activators in the regulation of rice seed expression genes. Plant Physiol. 2006;141:1694-707.

20. Li D, Yang C, Li X, Gan Q, Zhao X, Zhu L. Functional characterization of rice OsDof12. Planta. 2009;229:1159-69.

21. Wu Y, Messing J. Rapid divergence of prolamin gene promoters of maize after gene amplification and dispersal. Genetics. 2012;192:507-19.

22. Zhang Z, Zheng X, Yang J, Messing J, Wu Y. Maize endosperm-specific transcription factors $\mathrm{O} 2$ and PBF network the regulation of protein and starch synthesis. P Natl Acad Sci USA. 2016;113:10842.

23. Qi X, Li S, Zhu Y, Zhao Q, Zhu D, Yu J. ZmDof3, a maize endosperm-specific Dof protein gene, regulates starch accumulation and aleurone development in maize endosperm. Plant Mol Biol. 2017;93:1-14.

24. Yanagisawa S. Dof1 and Dof2 transcription factors are associated with expression of multiple genes involved in carbon metabolism in maize. Plant J. 2000;21:281-8

25. Corrales AR, Nebauer SG, Carrillo L, Fernández-Nohales P, Marqués J, RenauMorata B, Granell A, Pollmann S, Vicente-Carbajosa J, Molina RV, Medina J. Characterization of tomato Cycling Dof Factors reveals conserved and new functions in the control of flowering time and abiotic stress responses. J Exp Bot. 2014;65:995-1012.

26. Ravel C, Nagy IJ, Martre P, Sourdille P, Dardevet M, Balfourier F, Pont C, Giancola S, Praud S, Charmet G. Single nucleotide polymorphism, genetic mapping, and expression of genes coding for the DOF wheat prolamin-box binding factor. Funct Integr Genomic. 2006;6:310-21.

27. Shaw LM, Mclntyre CL, Gresshoff PM, Xue GP. Members of the Dof transcription factor family in Triticum aestivum are associated with lightmediated gene regulation. Funct Integr Genomic. 2009;9:485-98.

28. Dong GQ, Ni ZF, Yao YY, Nie XL, Sun QX. Wheat Dof transcription factor WPBF interacts with TaQM and activates transcription of an alpha-gliadin gene during wheat seed development. Plant Mol Biol. 2007;63:73-84.

29. Kumar R, Taware R, Gaur VS, Guru SK, Kumar A. Influence of nitrogen on the expression of TaDof1 transcription factor in wheat and its relationship with photosynthetic and ammonium assimilating efficiency. Mol Biol Rep. 2009; 36:2209-20.

30. Zhou J, Liu D, Deng X, Zhen S, Wang Z, Yan Y. Effects of water deficit on breadmaking quality and storage protein compositions in bread wheat (Triticum aestivum L.). J Sci Food Agric. 2018;98:4357-68.

31. Yanagisawa S, Izui K. Molecular cloning of two DNA binding proteins of maize that are structurally different but interact with the same sequence motif. J Biol Chem. 1993;268:16028-36.

32. Lijavetzky D, Carbonero P, Vicente-Carbajosa J. Genomewide comparative phylogenetic analysis of the rice and Arabidopsis Dof gene families. BMC Evol Biol. 2003;3:1-11.
33. Moreno-Risueno MA, Martinez M, Vicente-Carbajosa J, Carbonero P. The family of DOF transcription factors: from green unicellular algae to vascular plants. Mol Gen Genomics. 2007;277:379-90.

34. Kushwaha H, Gupta S, Singh VK, Rastogi S, Yadav D. Genome wide identification of Dof transcription factor gene family in sorghum and its comparative phylogenetic analysis with rice and Arabidopsis. Mol Biol Rep. 2011;38:5037-53.

35. Hernando-Amado S, González-Calle V, Carbonero P, Barrero-Sicilia C. The family of DOF transcription factors in Brachypodium distachyon: phylogenetic comparison with rice and barley DOFs and expression profiling. BMC Plant Biol. 2012;12:202.

36. Cai X, Zhang Y, Zhang C, Zhang T, Hu T, Ye J, Zhang J, Wang T, Li H, Ye Z. Genome-wide analysis of plant-specific Dof transcription factor family in tomato. J Integr Plant Biol. 2013;55:552-66.

37. Chen Y, Cao J. Comparative analysis of Dof transcription factor family in maize. Plant Mol Biol Report. 2015;33:1245-58.

38. Wen $\mathrm{CL}$, Cheng Q, Zhao L, Mao A, Yang J, Yu S, Weng Y, Xu Y. Identification and characterisation of Dof transcription factors in the cucumber genome. Sci Rep. 2016;6:23072.

39. Wu Z, Cheng J, Cui J, Xu X, Liang G, Luo X, Chen X, Tang X, Hu K, Qin C. Genomewide identification and expression profile of Dof transcription factor gene family in pepper (Capsicum annuum L.). front. Plant Sci. 2016;7:574.

40. Wei Q, Wang W, Hu T, Hu H, Mao W, Zhu Q, Bao C. Genome-wide identification and characterization of Dof transcription factors in eggplant (Solanum melongena L.). PeerJ. 2018;6:e4481.

41. International Wheat Genome Sequencing Consortium (IWGSC). Shifting the limits in wheat research and breeding using a fully annotated reference genome. Science. 2018;361:661.

42. Gu X. Statistical methods for testing functional divergence after gene duplication. Mol Biol Evol. 1999;16:1664-74.

43. Gaucher EA, Gu X, Miyamoto MM, Benner SA. Predicting functional divergence in protein evolution by site-specific rate shifts. Trends Biochem Sci. 2002;27:315-21.

44. Gu X. A simple statistical method for estimating type-II (cluster-specific) functional divergence of protein sequences. Mol Biol Evol. 2006;23:1937-45.

45. Yang Z. PAML 4: phylogenetic analysis by maximum likelihood. Mol Biol Evol. 2007;24:1586-91.

46. Fares MA, McNally D. CAPS: coevolution analysis using protein sequences. Bioinformatics. 2006;22: 2821-2.

47. Lescot M. PlantCARE, a database of plant cis-acting regulatory elements and a portal to tools for in silico analysis of promoter sequences. Nucleic Acids Res. 2002;30:325-7.

48. Koralewski TE, Krutovsky KV. Evolution of exon-intron structure and alternative splicing. PLoS One. 2011;6:e18055.

49. Malviya N, Gupta S, Singh VK, Yadav MK, Bisht NC, Sarangi BK, Yadav D. Genome wide in silico characterization of Dof gene families of pigeonpea (Cajanus cajan (L) Millsp). Mol Biol Rep. 2015;42:535-52.

50. Gupta S, Kushwaha H, Singh VK, Bisht NC, Sarangi BK, Yadav D. Genome wide in silico characterization of Dof transcription factor gene family of sugarcane and its comparative phylogenetic analysis with Arabidopsis, rice and sorghum. Sugar Tech. 2014;16:372-84.

51. Feldman M, Levy AA. Genome evolution due to allopolyploidization in wheat. Genetics. 2012;192:763-74.

52. Zhao Y, Ma R, Xu D, Bi H, Xia Z, Peng H. Genome-wide identification and analysis of the AP2 transcription factor gene family in wheat (Triticum aestivum L.). Frontiers in. Plant Sci. 2019;10:1286.

53. Ma J, Gao S, Stiller J, Jiang QT, Lan XJ, Liu YX, Zheng YL. Identification of genes bordering breakpoints of the pericentric inversions on $2 B, 4 B$, and $5 A$ in bread wheat (Triticum aestivum L.). Genome. 2015;58:385-90.

54. Gu X. Functional divergence in protein (family) sequence evolution. Genetica. 2003;118:133-41.

55. Fetterman CD, Rannala B, Walter MA. Identification and analysis of evolutionary selection pressures acting at the molecular level in five forkhead subfamilies. BMC Evol Biol. 2008;8:261.

56. Ma J, Li MY, Wang F, Tang J, Xiong AS. Genome-wide analysis of Dof family transcription factors and their responses to abiotic stresses in Chinese cabbage. BMC Genomics. 2015;16:33.

57. Song A, Gao T, Li P, Chen S, Guan Z, Wu D, Xin J, Fan Q, Zhao K, Chen F. Transcriptome-wide identification and expression profiling of the DOF transcription factor gene family in Chrysanthemum morifolium. Front Plant Sci. 2016;7:199. 
58. Lucas-Reina E, Romero-Campero FJ, Romero JM, Valverde F. An evolutionarily conserved DOF-CONSTANS module controls plant photoperiodic signaling. Plant Physiol. 2015;168:561-74.

59. Skirycz A, Radziejwoski A, Busch W, Hannah MA, Czeszejko J, Kwaśniewski M, Zanor MI, Lohmann JU, De Veylder L, Witt I, Mueller-Roeber B. The DOF transcription factor OBP1 is involved in cell cycle regulation in Arabidopsis thaliana. Plant J. 2008;56:779-92.

60. Konishi M, Yanagisawa S. Sequential activation of two Dof transcription factor gene promoters during vascular development in Arabidopsis thaliana. Plant Physiol Biochem. 2007:45:623-9.

61. Baumann K, De Paolis A, Costantino P, Gualberti G. The DNA binding site of the Dof protein NtBBF1 is essential for tissuespecific and auxin-regulated expression of the rolB oncogene in plants. Plant Cell. 1999;11:323-34.

62. Rock CD, Sun X. Crosstalk between ABA and auxin signaling pathways in roots of Arabidopsis thaliana (L.) Heynh. Planta. 2005:222:98-106.

63. Park JE, Park JY, Kim YS, Staswick PE, Jeon J, Yun J, Kim SY, Kim J, Lee YH, Park CM. GH3-mediated auxin homeostasis links growth regulation with stress adaptation response in Arabidopsis. J Biol Chem. 2007;282:10036-46.

64. Venkatesh J, Park SW. Genome-wide analysis and expression profiling of DNA-binding with one zinc finger (Dof) transcription factor family in potato. Plant Physiol Biochem. 2015:94:73-85.

65. Yuan HM, Liu WC, Jin Y, Lu YT. Role of ROS and auxin in plant response to metal-mediated stress. Plant Signal Behav. 2013;8:e24671.

66. Shahid M, Pourrut B, Dumat C, Nadeem M, Aslam M, Pinelli E. Heavymetalinduced reactive oxygen species: phytotoxicity and physicochemical changes in plants. Rev Environ Contam Toxicol. 2014;232:1-44.

67. Kurai T, Wakayama M, Abiko T, Yanagisawa S, Aoki N, Ohsugi R. Introduction of the $Z m D o f 1$ gene into rice enhances carbon and nitrogen assimilation under low-nitrogen conditions. Plant Biotechnol J. 2011;9:826-37.

68. Wang Y, Fu B, Pan L, Chen L, Fu X, Li K. Overexpression of Arabidopsis Dof1, GS1 and GS2 enhanced nitrogen assimilation in transgenic tobacco grown under low-nitrogen conditions. Plant Mol Biol Report. 2013;31:886-900.

69. Jin JP, Tian F, Yang DC, Meng YQ, Kong L, Luo JC, Gao G. PlantTFDB 4.0: toward a central hub for transcription factors and regulatory interactions in plants. Nucleic Acids Res. 2017:45:D1040-5.

70. Letunic I, Bork P. 20 years of the SMART protein domain annotation resource. Nucleic Acids Res. 2018;46:D493-6.

71. Finn RD, Coggill P, Eberhardt RY, Eddy SR, Mistry J, Mitchell AL, Potter SC, Punta M, Qureshi M, Sangrador-Vegas A, Salazar GA, Tate J, Bateman A. The Pfam protein families database: towards a more sustainable future. Nucleic Acids Res. 2016;44:D279-85.

72. Wang $M$, Yue $H$, Feng $K$, Deng P, Song W, Nie X. Genome-wide identification, phylogeny and expressional profiles of mitogen activated protein kinase kinase kinase (MAPKKK) gene family in bread wheat (Triticum aestivum L.). BMC Genomics. 2016;17:668.

73. Yin G, Xu H, Xiao S, Qin Y, Li Y, Yan Y, Hu Y. The large soybean (Glycine max) WRKY TF family expanded by segmental duplication events and subsequent divergent selection among subgroups. BMC Plant Biol. 2013;13:148.

74. Liu N, Dong L, Deng X, Liu Y, Liu D, Li M, Hu Y, Yan Y. Genome-wide identification, molecular evolution, and expression analysis of auxin response factor (ARF) gene family in Brachypodium distachyon L. BMC Plant Biol. 2018;18:336

75. Edgar RC. MUSCLE: a multiple sequence alignment method with reduced time and space complexity. BMC Bioinformatics. 2004;5:113.

76. Edgar RC. MUSCLE: multiple sequence alignment with high accuracy and high throughput. Nucleic Acids Res. 2004;32:1792-7.

77. Han Z, Liu Y, Deng X, Liu D, Liu Y, Hu Y, Yan Y. Genome-wide identification and expression analysis of expansin gene family in common wheat (Triticum aestivum, L.). BMC Genomics. 2019;20:101.

78. Hu B, Jin J, Guo AY, Zhang H, Luo J, Gao G. GSDS 2.0: an upgraded gene feature visualization server. Bioinformatics. 2015;31:1296-7.

79. Bailey $\mathrm{TL}$, Elkan C. Fitting a mixture model by expectation maximization to discover motifs in biopolymers. Proc Int Conf Intell Syst Mol Biol. 1994;2:2836.

80. Moore RC, Purugganan MD. The early stages of duplicate gene evolution. P Natl Acad Sci USA. 2003;100:15682-7.

81. Anisimova M, Bielawski JP, Yang Z. Accuracy and power of the likelihood ratio test in detecting adaptive molecular evolution. Mol Biol Evol. 2001;18: 1585-92.

82. Yang Z, Wong WS, Nielsen R. Bayes empirical bayes inference of amino acid sites under positive selection. Mol Biol Evol. 2005;22:1107-18.
83. Song W, Qin Y, Zhu Y, Yin G, Wu N, Li Y, Hu Y. Delineation of plant caleosin residues critical for functional divergence, positive selection and coevolution. BMC Evol Biol. 2014;14:124.

84. Kelley LA, Sternberg MJ. Protein structure prediction on the web: a case study using the Phyre server. Nat Protoc. 2009;4:363-471.

85. Kaur S, Dhugga KS, Beech R, Singh J. Genome-wide analysis of the cellulose synthase-like (Csl) gene family in bread wheat (Triticum aestivum L.). BMC Plant Biol. 2017;17:193.

86. Cao H, He M, Zhu C, Yuan L, Dong L, Bian Y, Zhang W, Yan Y. Distinct metabolic changes between wheat embryo and endosperm during grain development revealed by 2D-DIGE-based integrative proteome analysis. Proteomics. 2016;16:1515-36.

\section{Publisher's Note}

Springer Nature remains neutral with regard to jurisdictional claims in published maps and institutional affiliations.
Ready to submit your research? Choose BMC and benefit from:

- fast, convenient online submission

- thorough peer review by experienced researchers in your field

- rapid publication on acceptance

- support for research data, including large and complex data types

- gold Open Access which fosters wider collaboration and increased citations

- maximum visibility for your research: over $100 \mathrm{M}$ website views per year

At BMC, research is always in progress.

Learn more biomedcentral.com/submissions 